\title{
Weak turbulent approach to the wind-generated gravity sea waves
}

\author{
A. Pushkarev ${ }^{\text {a }}$, D. Resio $^{\text {b }}$, V. Zakharov ${ }^{\text {a,c,d,* }}$ \\ ${ }^{a}$ Waves and Solitons LLC, 918 W. Windsong Dr., Phoenix, AZ 85045, USA \\ ${ }^{\mathrm{b}}$ Coastal and Hydraulics Laboratory, US Army Engineer Research and Development Center, Halls Ferry Road, Vicksburg, MS 39180, USA \\ ${ }^{\mathrm{c}}$ Landau Institute for Theoretical Physics, Moscow 117334, Russia \\ d Department of Mathematics, University of Arizona, Tucson, AZ 85721, USA
}

\begin{abstract}
We performed numerical simulation of the kinetic equation describing behavior of an ensemble of random-phase, spatially homogeneous gravity waves on the surface of the infinitely deep ocean. Results of simulation support the theory of weak turbulence not only in its basic points, but also in many details. The weak turbulent theory predicts that the main physical processes taking place in the wave ensemble are down-shift of spectral peak and "leakage" of energy and momentum to the region of very small scales where they are lost due to local dissipative processes. Also, the spectrum of energy right behind the spectral peak should be close to the weak turbulent Kolmogorov spectrum which is the exact solution of the stationary kinetic (Hasselmann) equation. In a general case, this solution is anisotropic and is defined by two parameters-fluxes of energy and momentum to high wave numbers. Even in the anisotropic case the solution in the high wave number region is almost proportional to the universal form $\omega^{-4}$. This result should be robust with respect to change of the parameters of forcing and damping. In all our numerical experiments, the $\omega^{-4}$ Kolmogorov spectrum appears in very early stages and persists in both stationary and non-stationary stages of spectral development. A very important aspect of the simulations conducted here was the development of a quasi-stationary wave spectrum under wind forcing, in absence of any dissipation mechanism in the spectral peak region. This equilibrium is achieved in the spectral range behind the spectral peak due to compensation of wind forcing and leakage of energy and momentum to high wave numbers due to nonlinear four-wave interaction. Numerical simulation demonstrates slowing down of the shift of the spectral peak and formation of the bimodal angular distribution of energy in the agreement with field and laboratory experimental data. A more detailed comparison with the experiment can be done after developing of an upgraded code making possible to model a spatially inhomogeneous ocean.
\end{abstract}

(C) 2003 Elsevier B.V. All rights reserved.

PACS: 92.10.H; 05.45; 92.10.L; 42.65.H; K

Keywords: Weak turbulent theory; Kolmogorov spectrum; Quasi-stationary wave spectrum

\section{Introduction}

The phenomenon of wind-generated gravity waves on the sea surface is a very interesting object not only for oceanographers, naval architects and coastal engineers. It is also a subject of fundamental interest for physicists. The ocean waves are the most conspicuous natural example of weakly nonlinear waves in a strongly dispersing media.

* Corresponding author.

E-mail address: zakharov@math.arizona.edu (V. Zakharov). 
Indeed, on a deep water the dispersion relation is $\omega=\sqrt{g k}$, thus the dispersion is very strong. The level of nonlinearity could be measured by the characteristic steepness, $\mu=k a$ ( $k$ is an average wave number and $a$ is a wave amplitude). Numerous observational data show that typically, $\mu \simeq 0.1$ (see for instance [1]). Even in the condition of a strong storm, $\mu$ rarely exceeds this limit. Meanwhile, the critical steepness of the Stokes waves is $\mu \simeq 0.45$. Thus, the level of nonlinearity of the ocean waves is small or at least moderate. This statement is very much enhanced by the predictions of the weakly nonlinear statistical theory. According to this theory a characteristic time of evolution of the wave spectrum is

$$
\tau \simeq \frac{1}{\omega \mu^{4}}
$$

Even for $\mu \simeq 0.1$ this time is equal to $10^{4}$ wave periods.

The weakly nonlinear statistical ensemble of surface gravity waves can be described by the theory of weak turbulence. This theory is quite universal and is applicable to a very broad scope of physical phenomena, including waves in plasmas, waves in liquid super-fluid helium, Rossby waves, and acoustic waves. The references can be found in the monograph [2]. And this list is far from being complete.

The theory of weak turbulence is far advanced analytically. In this theory the evolution of basic correlation functions is described in terms of kinetic equations for the wave action. These kinetic equations are nothing but standard kinetic equations for bosons, traditionally used in statistical physics since 1920s. The new point is the following: we deal now not with thermodynamically equilibrium solutions, which are not relevant for description of a real wave turbulence, but focus our interest on Kolmogorov-type solutions. These solutions carry a finite amount of constants of motion (energy, momentum, wave numbers) from the region in $k$-space, where they are generated, to the region where they are accumulated or absorbed by some kind of dissipation mechanism. In the theory of weak turbulence we study these equations in the limit of very high occupation numbers, where the equations become homogeneous with respect to the distribution function (quadratic, cubic, etc). As a result, in most physical situations the Kolmogorov-type solutions are power-like functions.

The analytical theory of weak turbulent Kolmogorov solutions has been studied in detail, but the experimental and the numerical justifications of this theory cannot be considered as being sufficient. There is only one physical situation, the capillary wave turbulence, where the weak turbulent theory is strongly supported by the experiment and the numerical simulation [3-5].

It is extremely challenging and attractive to apply the theory of weak turbulence to such a great natural laboratory as the world ocean. In this paper we make a step in this direction. We present here our numerical experiments on the solution of the Hasselmann's kinetic equation for gravity waves on a deep sea. We show that these experiments completely confirm the prediction of the wave turbulent theory. First of all, they confirm the fundamental role of the universal Kolmogorov spectrum $\varepsilon_{\omega} \simeq \omega^{-4}$, which was found by Zakharov and Filonenko in 1966 [6]. They make it possible to explain in a natural way a lot of experimental data accumulated in the physical oceanography for decades.

\section{General consideration}

Let $\eta(\vec{r}, t)$ be a surface elevation, $\psi(\vec{r}, t)$ be a potential on the surface. We assume that density of the fluid $\rho=1$. The complex amplitude of propagating waves is given by the formula:

$$
a_{k}=\frac{1}{\sqrt{2}}\left[\left(\frac{g}{k}\right)^{1 / 4} \eta_{k}-\mathrm{i}\left(\frac{k}{g}\right)^{1 / 4} \psi_{k}\right] .
$$


In the pair of correlation functions,

$$
\left\langle a_{k} a_{k^{\prime}}^{*}\right\rangle=g N_{k} \delta_{k-k^{\prime}},
$$

where $N_{k}$ is a spectral density of the wave action. This definition of wave action is common in oceanography. It has dimension $N_{k} \sim L^{4} T$. The Hamiltonian describing the motion of fluid is a functional that includes terms of all orders in expansion on $a_{k}, a_{k}^{*}$. One can perform the canonical transformation to new variables $b_{k}$, excluding the cubic terms in the Hamiltonian. For new variables $b_{k}$ we have

$$
\left\langle b_{k} b_{k^{\prime}}^{*}\right\rangle=g n_{k} \delta_{k-k^{\prime}} .
$$

The complex amplitude $a_{k}$ is expressed through $b_{k}$ as the power series, as well as $N_{k}$ through $n_{k}$. Their cumbersome coefficients are presented in Appendix A (see also [33]). The difference between $N_{k}$ and $n_{k}$ on a deep water is of the order $\mu^{2}$, and can be neglected. In shallow water this difference is much more important.

If the nonlinearity is weak, the fluid is described by the Hamiltonian

$$
H=\int \omega_{k} b_{k} b_{k}^{*} \mathrm{~d} k+\frac{1}{4} \int T_{k k_{1} k_{2} k_{3}} b_{k}^{*} b_{k_{1}}^{*} b_{k_{2}} b_{k_{3}} \delta_{k+k_{1}-k_{2}-k_{3}} \mathrm{~d} k \mathrm{~d} k_{1} \mathrm{~d} k_{2} \mathrm{~d} k_{3} .
$$

Here $T$ is a homogeneous function of third order,

$$
T\left(\epsilon k, \epsilon k_{1}, \epsilon k_{2}, \epsilon k_{3}\right)=\epsilon^{3} T\left(k, k_{1}, k_{2}, k_{3}\right) .
$$

The explicit formula for $T_{k k_{1} k_{2} k_{3}}$ is in Appendix B.

The kinetic equation for $n_{k}$ reads

$$
\frac{\partial n_{k}}{\partial t}=S_{\mathrm{nl}}+\gamma(k) n_{k} .
$$

This equation was derived by Hasselmann in 1962 [7,8], and broadly applied in oceanography. Hasselmann erroneously considered that Eq. (2.4) is written for $N_{k}$, and this view is shared until now by most oceanographers. While the difference between $n_{k}$ and $N_{k}$ is relatively small for deep water, it becomes significant for shallow water.

In $(2.4)$

$$
\begin{aligned}
S_{\mathrm{nl}}=2 \pi g^{2} & \int_{\left|k_{2}\right|<\left|k_{3}\right|}\left|T_{k k_{1} k_{2} k_{3}}\right|^{2}\left(n_{k_{1}} n_{k_{2}} n_{k_{3}}+n_{k} n_{k_{2}} n_{k_{3}}-n_{k} n_{k_{1}} n_{k_{2}}-n_{k} n_{k_{1}} n_{k_{3}}\right) \delta\left(\omega_{k}+\omega_{k_{1}}-\omega_{k_{2}}-\omega_{k_{3}}\right) \\
& \times \delta\left(\vec{k}+\vec{k}_{1}-\vec{k}_{2}-\vec{k}_{3}\right) \mathrm{d} k_{1} \mathrm{~d} k_{2} \mathrm{~d} k_{3} .
\end{aligned}
$$

The function $\gamma_{k}$ describes the active forcing by the wind and the damping due to the wave breaking. Due to complexity of these processes, this function should be found from the experiments. For large and moderate scales $\gamma_{k}$ is dominated by the interaction with the wind. Due to the large difference between air and water densities, $\gamma_{k}$ is small and is of order

$$
\frac{\gamma_{k}}{\omega_{k}} \simeq \epsilon \sim \frac{\rho_{\text {air }}}{\rho_{\text {water }}} \text {. }
$$

From (2.4) one obtains $\mu \simeq \epsilon^{1 / 4}$, in accordance with experimental data. For the rate of the spectrum evolution one has

$$
\omega \tau \simeq \frac{1}{\epsilon} \sim 10^{3}
$$

Applicability of the weak turbulent theory in a final degree comes from the small value of $\epsilon$. 
Hereafter the polar coordinates $\omega=\sqrt{g k}, \theta$ in $k$-plane are used. The wind velocity $V$ defines the characteristic frequency $\omega_{0}=g / V$. Even for weak wind, $V \simeq 1-2 \mathrm{~m} / \mathrm{s}$, characteristic frequency is by the order of magnitude less than the frequency $\omega_{\mathrm{c}} \simeq 30 \mathrm{~Hz}$, where the effects of capillarity become important. For $\omega<\omega_{0}, \gamma$ is negative, small and unknown. It is defined by friction between sea surface and turbulent air boundary layer. For $\omega>\omega_{0}, \gamma$ is positive due to Cherenkov-type excitation of waves by the wind. According to Donelan et al. [9], one can put

$$
\gamma(\omega, \theta)= \begin{cases}0.2 \epsilon\left(\frac{\omega}{\omega_{0}}-1\right)^{2} \omega \cos \theta, & \cos \theta>0, \omega>\omega_{0}, \\ 0 & \text { otherwise. }\end{cases}
$$

This expression can be trusted up to $\omega$ equal to 5-6 $\omega_{0}$. For higher frequencies experimental data are scarce, and the expression for $\gamma(\omega, \theta)$ is not clearly known.

If the wind is weak enough, $U<5 \mathrm{~m} / \mathrm{s}$, a wave breaking is absent, the sea surface is smooth, and $\gamma>0$ at least up to $\omega \simeq \omega_{\text {cap }}$. For stronger winds, the effects of micro-scale and macro-scale (white capping) wave breaking make $\gamma<0$ in the high enough frequency region (see [10]). In both cases there is an effective sink of wave energy in small scales. In the absence of wind velocity, this sink is realized either by excitation of capillary waves and their viscous dissipation, or by the wave breaking.

Existence of this sink leads to a conjecture that real physics of wind-driven waves on the sea surface can be compared with the physics of turbulence in the incompressible fluid at high Reynolds numbers.

It is well known that kinetic equation (2.4), if $\gamma=0$, has constants of motion. In the isotropic case they are

$$
E=\int \omega_{k} n_{k} \mathrm{~d} k
$$

and a wave action

$$
N=\int n_{k} \mathrm{~d} k
$$

In the general case it also preserves momentum

$$
\vec{R}=\int \vec{k} n_{k} \mathrm{~d} k
$$

In reality neither energy nor momentum are the constants of motion. They "leak" to the region of high wave numbers (similar situation takes place in turbulence of incompressible fluid). Only wave action is the true constant of motion. Other conservation laws (of energy and momentum) are just formal. The problem of non-conservation of formal motion constants is discussed in detail in the paper of Pushkarev and Zakharov [15].

The effect of the "leakage" of energy to high wave numbers is clearly demonstrated practically by all numerical experiments of the Hasselmann equation, since pioneering works of Hasselmann et al. [16]. In a typical case the angle-averaged $S_{\mathrm{nl}}$

$$
f(\omega)=\frac{1}{2 \pi} \int_{0}^{2 \pi} S_{\mathrm{nl}} \mathrm{d} \theta
$$

is a "two-lobe" function. It has only one zero at $\omega=\omega_{p}$ and $f(\omega)>0$ for $0<\omega<\omega_{p}$, while $f(\omega)<0$ for $\omega>\omega_{p}$. Preservation of both wave action and energy means that $f(\omega)$ satisfies simultaneously two conditions:

$$
\begin{aligned}
& \int_{0}^{\infty} \omega^{3} f(\omega) \mathrm{d} \omega=0, \\
& \int_{0}^{\infty} \omega^{4} f(\omega) \mathrm{d} \omega=0 .
\end{aligned}
$$


Apparently, it is impossible if $f(\omega)$ is a "two-lobe" function. Integral (2.13) must be negative. We denote

$$
\int_{0}^{\infty} \omega^{4} f(\omega) \mathrm{d} \omega=-g^{2} \frac{P}{2} .
$$

Here $P$ is the flux of energy to high wave numbers. The inevitable presence of the flux lead us to a theory of Kolmogorov style.

In the Kolmogorov theory of turbulence the spectra are governed by fluxes of the constants of motion. Due to the presence of two constants of motion, even in the isotropic case, the turbulence of gravity waves is qualitatively similar to turbulence of two-dimensional incompressible fluid, which is governed by fluxes of energy and enstrophy.

We should stress here the fundamental difference between weak (wave) and strong (hydrodynamic) turbulence. In the theory of turbulence Kolmogorov spectra are just a plausible hypothesis, which is not supported properly by rigorous arguments. In the theory of weak turbulence, Kolmogorov spectra appear as exact solution of the equation

$$
S_{\mathrm{nl}}=0 .
$$

For gravity waves on the surface of a fluid the most important Kolmogorov spectrum, describing the direct cascade of energy to high frequencies has a form

$$
\varepsilon_{\omega} \simeq P^{1 / 3} \omega^{-4} .
$$

In 1966 Zakharov and Filonenko found that spectrum (2.16) satisfies Eq. (2.15). In 1972 spectra with this form were experimentally observed by Toba [11], who was not aware of the work of Zakharov and Filonenko. The interpretation of spectrum (2.16) as a Kolmogorov spectrum was published first in 1982 by Zakharov and Zaslavskii [12] and then propagated by Kitaigorodskii [13].

\section{Weak turbulent Kolmogorov spectra}

In this section we summarize the basic facts on weakly turbulent Kolmogorov spectra. We discuss solutions of Eq. (2.15) and present these facts without detailed analytical justification. This justification is referred to Appendix C in a brief form.

Naively, one can think that this equation has thermodynamic solutions of the form

$$
n_{k}=\frac{T}{\omega_{k}+C} \text {. }
$$

In fact, in the considered case of gravity waves these solutions do not exist because of divergence of the integral (2.5) at large wave numbers. Let us call a function $n_{k}$ "allowed" if the integrals in the operator $S_{\mathrm{nl}}\left[n_{k}\right]$ are converged for both $k \rightarrow \infty$ and $k \rightarrow 0$.

To determine the class of allowed functions one put $k_{1} \rightarrow \infty, k_{3} \rightarrow \infty$. From the conditions

$$
\begin{aligned}
& \vec{k}+\vec{k}_{1}=\vec{k}_{2}+\vec{k}_{3}, \\
& \omega_{k}+\omega_{k_{1}}=\omega_{k_{2}}+\omega_{k_{3}},
\end{aligned}
$$

one can see that at $\vec{k}_{1} \rightarrow \infty, \vec{k}_{3} \rightarrow \infty, k_{2}$ remains finite $\left(\left|k_{2}\right| \sim|k|\right)$.

The contribution $S_{\mathrm{nl}}^{(1)}$ to the integral (2.5) comes from integration over large $k_{1}$, and can be written approximately as follows:

$$
S_{\mathrm{nl}}^{(1)} \simeq 2 \pi g^{2} n_{k} \int\left|T_{k k_{1}}\right|^{2} n_{k_{2}} \delta\left(\omega_{k}-\omega_{k_{2}}\right)\left(\vec{k}-\vec{k}_{2}, \frac{\partial n}{\partial \vec{k}_{1}}\right) \mathrm{d} \vec{k}_{1} \mathrm{~d} \vec{k}_{2},
$$




$$
T_{k k_{1}}=T_{k k_{1}, k k_{2}},
$$

where $(\cdot, \cdot)$ means scalar product.

As far as (see Appendix B) $\left|T_{k k_{1}}\right|^{2} \simeq k_{1}^{2} k^{4}$ at $k_{1} \gg k$, integral (3.4) converges if

$$
n_{k}<\frac{C}{k^{3}}
$$

at $k \rightarrow \infty$. Thermodynamic solutions do not satisfy the condition (3.6).

Let $k_{1} \rightarrow 0$. Due to (3.2) $k_{2} \rightarrow 0$ as well. The contribution $S_{\mathrm{nl}}^{(2)}$ provided by integration over small $k_{1}, k_{2}$ reads

$$
\begin{aligned}
S_{\mathrm{nl}}^{(2)} \simeq 2 \pi g^{2} \int & n_{k_{1}} n_{k_{2}}\left\{\left|T_{k, k_{1}, k_{2}, k+k_{1}-k_{2}}\right|^{2}\left(n_{k+k_{1}-k_{2}}-n_{k}\right) \delta\left(\omega_{k}+\omega_{k_{1}}-\omega_{k+k_{1}-k_{2}}-\omega_{k_{2}}\right)\right. \\
& \left.+\left|T_{k, k_{2}, k_{1}, k+k_{2}-k_{1}}\right|^{2}\left(n_{k-k_{1}+k_{2}}-n_{k}\right) \delta\left(\omega_{k}+\omega_{k_{2}}-\omega_{k-k_{1}+k_{2}}-\omega_{k_{1}}\right)\right\} \mathrm{d} k_{1} \mathrm{~d} k_{2} .
\end{aligned}
$$

The integrand should be expanded in Taylor series over $k_{1}, k_{2}$. The first term of the expansion vanishes due to the symmetry. In the second approximation kinetic equation transforms to the diffusion equation

$$
\begin{aligned}
& \frac{\partial n}{\partial t}=\operatorname{div} D(k) \nabla n, \\
& D(k)=\frac{\pi}{2} g^{2} \int\left|T_{k k_{1}}\right|^{2} n_{k} n_{k_{1}}\left(k_{1}-k_{2}\right)^{2} \delta\left(\omega_{k_{1}}-\omega_{k_{2}}\right) \mathrm{d} k_{1} \mathrm{~d} k_{2} .
\end{aligned}
$$

Suppose that $n_{k} \simeq k^{-s}$. Integral (3.9) converges if $s<19 / 4$. Thus $n_{k}$ must satisfy the condition

$$
n_{k}<\frac{C}{k^{19 / 4}}, \quad k \rightarrow 0 .
$$

Conditions (3.6) and (3.10) define the class of allowed functions. In particular, the power-like function $n_{k}=k^{-x}$ is allowed if:

$$
3<x<\frac{19}{4} \text {. }
$$

Let us formulate the central results of the theory of weak turbulence. Suppose that an ensemble of weakly nonlinear waves in the space of dimension $d$ is described by kinetic equation (2.4). Suppose that the following conditions are satisfied:

1. Eq. (2.4) is invariant with respect to rotations in $d$-dimensional $k$-space. This condition implies that the dispersion law depends only on the modulus of $k: \omega=\omega(|k|)$.

2. There is no characteristic length in the system. It implies that $\omega$ is a power-like function, while $T$ is a homogeneous function of its arguments $\omega=|k|^{\alpha}$ :

$$
T\left(\epsilon k, \epsilon k_{1}, \epsilon k_{2}, \epsilon k_{3}\right)=\epsilon^{\beta} T\left(k, k_{1}, k_{2}, k_{3}\right) .
$$

In this case equation (2.15) has no more than four power-like solutions:

$$
\begin{aligned}
& n_{k}=k^{-x_{i}}, \quad i=1, \ldots, 4, \\
& x_{1}=\frac{2}{3} \beta+d, \quad x_{2}=\frac{1}{3}(2 \beta-\alpha)+d, \quad x_{3}=\alpha, \quad x_{4}=0 .
\end{aligned}
$$

The solutions are

$$
n_{1} \sim|k|^{-(2 \beta / 3)-d},
$$




$$
\begin{aligned}
& n_{2} \sim|k|^{-((2 \beta-\alpha) / 3)-d}, \\
& n_{3} \sim \frac{T}{k^{\alpha}}, \\
& n_{4} \sim \lambda=\text { const., } \quad \lambda=\lim _{\substack{T \rightarrow \infty \\
\mu \rightarrow \infty}} \frac{T}{\mu} .
\end{aligned}
$$

To find a real amount of power-like solutions, one should determine the class of functions, allowed by the $S_{\mathrm{nl}}$. If this class does not include power-like functions, neither of solutions (3.15)-(3.18) is relevant for description of a real physical simulation. Suppose that power-like functions $n_{k}=k^{-x}$ are allowed if

$$
s_{1}<x<s_{2} .
$$

The power-like solution $n_{k} \simeq k^{-x_{i}}$ is physically relevant if $x_{i}$ belongs to this interval

$$
s_{1}<x_{i}<s_{2} .
$$

In the case of gravity waves on deep water the conditions (3.6) and (3.10) are obviously satisfied and $\alpha=1 / 2$, $\beta=3$. Hence

$$
x_{1}=4, \quad x_{2}=\frac{23}{6}, \quad x_{3}=\frac{1}{2}, \quad x_{4}=0 .
$$

According to (3.6) and (3.10) $s_{1}=3, s_{2}=19 / 4$. One can see that $s_{1}<x_{1}<s_{2}$ and $s_{1}<x_{2}<s_{2}$, while $x_{3}<s_{1}$, $x_{4}<s_{1}$. Hence, the only solutions which can be used for description of real physical situations are the solutions corresponding to $x_{1}$ and $x_{2}$. These solutions are weak turbulent Kolmogorov spectra. We define spectral density of energy by relation

$$
\varepsilon_{\omega} \mathrm{d} \omega=\omega(k) n(k) \mathrm{d} \vec{k}=\frac{2 \omega^{4}}{g^{2}} n(\omega, \theta) \mathrm{d} \omega \mathrm{d} \theta
$$

In terms of energy density Kolmogorov spectra read:

$$
\begin{aligned}
& \varepsilon_{\omega}^{(1)}=C_{0} g^{4 / 3} P^{1 / 3} \omega^{-4}, \\
& \varepsilon_{\omega}^{(2)}=q_{0} g^{4 / 3} Q^{1 / 3} \omega^{-11 / 3} .
\end{aligned}
$$

In (3.23) and (3.24), $P$ is the energy flux to high wave numbers, $Q$ is the wave action flux to small wave numbers. $C_{0}$ and $q_{0}$ are dimensionless Kolmogorov constants.

According to (3.22)

$$
\begin{aligned}
& n_{k}^{(1)}=\frac{C_{0}}{2 g^{2 / 3}} P^{1 / 3} k^{-4}, \\
& n_{k}^{(2)}=\frac{q_{0}}{2 g^{1 / 2}} Q^{1 / 3} k^{-23 / 6} .
\end{aligned}
$$

From (2.1) one obtains

$$
\eta_{k}=\frac{1}{\sqrt{2}}\left(\frac{k}{g}\right)^{1 / 4}\left(a_{k}+a_{-k}^{*}\right)
$$

Hence

$$
I_{k}=\left\langle\left|\eta_{k}\right|^{2}\right\rangle=\frac{1}{2}(g k)^{1 / 2}\left(N_{k}+N_{-k}\right) .
$$


Here $I_{k}=I_{-k}$ is the spatial spectrum. For deep water one can neglect the difference between $N_{k}$ and $n_{k}$ and put according to (3.25), (3.26) and (3.28)

$$
\begin{aligned}
& I_{k}^{(1)}=\frac{C_{0}}{2} \frac{g^{3 / 2} P^{1 / 3}}{k^{7 / 2}}, \\
& I_{k}^{(2)}=\frac{q_{0}}{2} \frac{g^{3 / 2} Q^{1 / 3}}{k^{10 / 3}} .
\end{aligned}
$$

Power-like isotropic Kolmogorov spectra are not unique solutions of Eq. (2.15). One has to expect that this equation has also an anisotropic power-like Kolmogorov spectrum

$$
\varepsilon_{\omega}^{(3)}=M^{1 / 3} f(\theta) \omega^{-13 / 3} g^{5 / 3} .
$$

Here $M$ is the flux of momentum along the $x$-axis to high-frequency region. In (3.31) $f(\theta)$ is an unknown function of the angle with respect to the real axis which cannot be found analytically in a general case. It can be done for a special "diffusion" model (see [14]).

Moreover, from the symmetry consideration one has

$$
f(-\theta)=-f(\theta)
$$

hence his function is not positively defined and cannot be a model of any real spectrum.

More general Kolmogorov-type solutions are governed by more than one flux of motion constants. Even in the isotropic case a general solution of (2.15) must have a form

$$
\varepsilon_{\omega}=\frac{g^{4 / 3} P^{1 / 3}}{\omega^{4}} F\left(\frac{\omega Q}{P}\right)
$$

where $F(\xi)$ is some unknown positive function, satisfying the conditions $\xi=\omega Q / P$. Here $P$ is the flux of energy originated by sources concentrated at $k \rightarrow \infty, Q$ is the flux of wave action, coming from infinity.

Then

$$
\begin{aligned}
& F(0)=C_{0}, \\
& F(\xi) \rightarrow q_{0} \xi^{1 / 3}, \quad \xi \rightarrow \infty .
\end{aligned}
$$

Spectrum (3.33) describes the situation when there is the source of energy $P$ in small frequencies and source of wave action $Q$ in high frequencies.

The most general Kolmogorov solution of the equation $S_{\mathrm{nl}}=0$ has the form

$$
\varepsilon_{\omega}=\frac{g^{4 / 3} P^{1 / 3}}{\omega^{4}} G\left(\frac{\omega Q}{P}, \frac{g M}{\omega P}, \theta\right) .
$$

Here $G$ is some function of three variables to be found numerically by solution of the system of nonlinear integral equations imposed on the Fourier component of angular-frequency spectrum (see Appendix C). We plan to undertake a full-scale numerical experiment for definition of this function. Some particular properties of this function, however, can be found analytically.

General Kolmogorov spectrum (3.36) appears in the case when one has sources of energy and momentum $P, M$ at small wave numbers together with the source of wave action $Q$ at high wave numbers. In the situation we are discussing (direct cascade) there is no flux of wave action from infinity, and $Q=0$. In this case one has

$$
\varepsilon_{\omega}=\frac{g^{4 / 3} P^{1 / 3}}{\omega^{4}} H\left(\frac{g M}{\omega P}, \theta\right)
$$


Let us introduce dimensionless parameter $\xi=g M / \omega P$. For completely isotropic spectrum $M=0$, hence $\xi=0$. One can say that the value of $\xi$ characterizes the degree of anisotropy. For small values of $\xi$ function $H$ can be expanded in a Taylor series

$$
H=H_{0}(\theta)+H_{1}(\theta) \xi+\cdots .
$$

Apparently, $H_{0}(\theta)=C_{0}$ does not depend on $\theta$. This is just the Kolmogorov constant, introduced in (3.23). One can prove that $H_{1}(\theta)=C_{1} \cos \theta[2]$.

The constants $C_{0}, C_{1}$ can be called the first and and the second Kolmogorov constants. We established that for small $\xi$

$$
\varepsilon_{\omega}=\frac{g^{4 / 3} P^{1 / 3}}{\omega^{4}}\left(C_{0}+C_{1} \frac{g M}{\omega P} \cos \theta+\cdots\right)
$$

This case is realized at any values of $M, P$ if $\omega \rightarrow \infty$. Hence the spectrum (3.36) becomes completely isotropic at large values of $\omega$.

One can determine $H(\xi, \theta)$ at very large values of $\xi$. In this "extremely" anisotropic case the spectrum is governed by a single parameter $M$ and its dependence on the flux of energy $P$ should drop out. It means that in this limit $H(\xi, \theta) \rightarrow \xi^{1 / 3} f(\theta)$ at $\xi \rightarrow \infty$ and formula (3.37) goes to the formula (3.31).

In reality the simple formula (3.39) gives a reasonable approximation to observed spectra. Banner [1] found, by analysis of the experimental data, that the averaged by angles spectrum behave like $\omega^{-4}$, while the one-dimensional slice at $\theta=0$ goes to zero faster. Banner assumes that its behavior obeys the Phillips law $\omega^{-5}$. Another words, according to Banner

$$
\frac{\varepsilon_{\omega}(0)}{\left\langle\varepsilon_{\omega}\right\rangle} \simeq \frac{1}{\omega} \text {. }
$$

According to our formula (3.39)

$$
\frac{\varepsilon_{\omega}(0)}{\left\langle\varepsilon_{\omega}\right\rangle} \simeq C_{0}+\frac{C_{1} M}{P \omega} .
$$

This is the decreasing function of $\omega$ as well and our results coincide with Banner's results at least on a qualitative level. However, the difference due to presence of constant $C_{0}$ in our formula (3.41) is very essential. $C_{0}$ is the Kolmogorov constant, which certainly cannot be zero.

\section{Matching with sources and non-stationary behavior}

Now we discuss under what conditions weak turbulent Kolmogorov spectra can be realized in a physical situation. We will discuss only the "direct cascade", which is described in a general anisotropic case by the spectrum (3.37). First and foremost condition for realization of this spectrum is an efficient sink in the high-frequency domain. For surface waves this sink is provided by generation of capillary waves or wave breaking. In the framework of the model (2.4) the sink is described by $\gamma(k)<0$ at $|k|>k_{d}, \gamma(k) \rightarrow-\infty$ if $|k| \rightarrow \infty$.

Like in the Kolmogorov theory of turbulence in incompressible fluid, a detailed shape of $\gamma(k)$ is not important. Damping coefficient $\gamma(k)$ just must absorb fluxes of energy and momentum coming from the small frequency region. In the conditions of full absorption

$$
P=-\int_{|k|>k_{d}} \gamma(k) \omega(k) n(k) \mathrm{d} k
$$




$$
M=-\int_{|k|>k_{d}} \gamma(k) k n(k) \mathrm{d} k .
$$

The ideal conditions for realization of Kolmogorov spectrum (3.37) takes place if the region of instability, where $\gamma(k)>0$, is localized in the domain

$$
k_{0}<k<k_{1}
$$

and $k_{1} \ll k_{d}$. To provide absorption of the inverse cascade which is forming at $k<k_{0}$, one should have damping at $k<k_{0}$. Thus $\gamma(k)<0$ at $k<k_{0}$.

In this situation one can expect formation of a stationary spectrum, obeying the equation

$$
S_{\mathrm{nl}}+\gamma(k) n_{k}=0 .
$$

A shape of the spectrum in the region $0<k<k_{1}$ cannot be predicted from the general principles. But in the "window of transparency"

$$
k_{1}<k<k_{d}
$$

one expects the appearance of a Kolmogorov spectrum (3.37), defined by the fluxes $P, M$.

By integrating (4.4) one has

$$
\begin{aligned}
& P=\int_{|\vec{k}|<k_{1}} \omega_{k} \gamma_{k} n_{k} \mathrm{~d} \vec{k}, \\
& P=-\int_{|\vec{k}|<k_{1}} \omega_{k} S_{\mathrm{nl}} \mathrm{d} \vec{k} .
\end{aligned}
$$

In a similar way

$$
\begin{aligned}
& M=\int_{|\vec{k}|<k_{1}} \gamma_{k} k \cos \theta n_{k} \mathrm{~d} \vec{k}, \\
& M=-\int_{|\vec{k}|<k_{1}} k \cos \theta S_{\mathrm{nl}} \mathrm{d} \vec{k} .
\end{aligned}
$$

Thus we have three different ways for calculation of the fluxes $P, M$.

We want to point out that localization of instability in small wave number is the sufficient, but not the necessary condition for forming of the Kolmogorov spectrum of the inverse cascade. The income of energy $\varepsilon^{+}$, defined by the formula

$$
\varepsilon_{k}^{+}=\gamma(k) \omega(k) n(k)
$$

includes product of $\gamma(k)$ and $n(k)$. Even if $\gamma(k)$ grows at large $k$, the product $\gamma(k) \omega(k) n(k)$ could be concentrated at small $k$.

Let us suppose that there is no damping in small $k$. In this case no stationary state can be established. In the region $k<k_{0}$ one will observe formation of the inverse cascade, propagating of a front toward $k \rightarrow 0$. Meanwhile, in $k \geq k_{0}$ a stationary state will be reached in a finite time. Formulae (4.1) and (4.2) as well as (4.7)-(4.9) remain valid, while formulae (4.6) and (4.8) are not correct anymore.

Propagation of the inverse cascade front is described by the self-similar solution of the equation

$$
\frac{\partial n}{\partial t}=S_{\mathrm{nl}} .
$$


It has the following self-similar solution

$$
n=t^{\alpha} U\left(k t^{\beta}\right)
$$

where $\alpha$ and $\beta$ are connected by the relation

$$
2 \alpha+1=\frac{19}{2} \beta .
$$

To determine $\alpha, \beta$ one should use some additional information about the solution. In the case of inverse cascade this information can be extracted from the fact that there is no flux of wave action to high frequencies if $k_{1} \ll k_{d}$. All gained wave action is deposited to the wave ensemble. Assuming that in the instability region $k \sim k_{0}$ the stationary state is reached, one has

$$
N=\int n_{k} \mathrm{~d} k \sim t
$$

Hence

$$
\alpha-2 \beta=1, \quad \beta=\frac{6}{11}, \quad \alpha=\frac{23}{11} .
$$

Solution (4.12) takes a form

$$
n=t^{23 / 11} U\left(k t^{6 / 11}\right)
$$

and the front propagates to small $k$ according to the law

$$
k_{\mathrm{f}} \sim t^{-6 / 11} .
$$

The region $k \sim 0$ has "infinite capacity" and can absorb infinite amount of wave energy.

At $k \simeq k_{1}$ solution (4.12) should be matched with the Kolmogorov spectrum (3.37) with some fluxes $P, M$ which are formed in the instability region.

Another important self-similar solution describes the evolution of "swell" or water waves in the absence of any type of sources. In this case wave action is preserved, while energy and momentum leak to $k \rightarrow \infty$. Preservation of $N$ implies

$$
\alpha=2 \beta, \quad \beta=\frac{2}{11}, \quad \alpha=\frac{4}{11} .
$$

The self-similar solution has the form

$$
n=t^{4 / 11} U\left(k t^{2 / 11}\right) \text {. }
$$

It describes down-shift of wave maximum

$$
k_{\mathrm{m}} \simeq t^{-2 / 11} .
$$

Total energy and momentum of solution (4.19) decreases as

$$
\varepsilon \simeq t^{-1 / 11}, \quad M \simeq t^{-2 / 11} .
$$

Finally we discuss a self-similar solution describing formation of direct cascade Kolmogorov spectrum. An additional constrain on $\alpha, \beta$ can be found from the assumption that at small $k$ Kolmogorov spectrum is already established. In this area $n$ does not depend on time, and $U \simeq \xi^{-4} \sim 1 / k^{4}$. It might happen only if

$$
\alpha=4 \beta, \quad \beta=\frac{2}{3}, \quad \alpha=\frac{8}{3} .
$$


The corresponding solution has a form

$$
n=\left(t_{0}-t\right)^{8 / 3} U\left(k\left(t_{0}-t\right)^{2 / 3}\right) .
$$

Formula (4.23) describes propagation of the "shock" wave to the high-frequency region. A trajectory of the shock

$$
k_{M} \simeq \frac{1}{\left(t-t_{0}\right)^{2 / 3}} .
$$

This shock is self-accelerating. It reaches infinity in a finite time and Kolmogorov spectrum $\omega^{-4}$ is established in an explosive way.

Finally, let us perform an elementary derivation of the Kolmogorov spectrum $\omega^{-4}$. To do this, we return to the stationary equation (4.4). Let $k_{s}$ be some wave number inside the interval of transparency $k_{1}<k_{s}<k_{d}$. Multiplying (4.4) by $\omega_{k}$ and integrating by the domain $|k|<k_{s}$, one find for the local value of energy flux

$$
P\left(k_{s}\right)=\int_{|k|<k_{s}} \omega_{k} S_{\mathrm{nl}} \mathrm{d} k
$$

On the other hand, $\gamma_{k}=0$ if $k_{1}<k<k_{d}$. Hence, $P$ is defined by the formulae (4.6) and (4.7).

Let us assume that

$$
n_{k}=C_{0} P^{1 / 3} k^{-x}, \quad 3<x<\frac{19}{4} .
$$

Plugging (4.26) into (4.25) and taking into account convergence of the operator $S_{\mathrm{nl}}$ on this class of allowed functions, one finds from a dimension consideration

$$
P\left(k_{s}\right)=P C_{0}^{3} \lambda k_{s}^{12-3 x} .
$$

As far as the flux $P\left(k_{s}\right)$ does not depend on $k_{s}$, one find

$$
\begin{aligned}
& x=4, \\
& C_{0}=\lambda^{-(1 / 3)} \neq 0 .
\end{aligned}
$$

Comparing with (3.22) one can see that we obtained again Zakharov-Filonenko spectrum $\varepsilon \simeq \omega^{4}$. Due to convergence of $S_{\mathrm{nl}}, \lambda$ is finite and $C_{0} \neq 0$.

Note that for the Phillips spectrum $\varepsilon_{\omega} \simeq \omega^{-5}$ gives $\lambda=9 / 2$. In this case

$$
P\left(k_{s}\right) \simeq k_{s}^{-3 / 2},
$$

and

$$
P\left(k_{s}\right) \rightarrow 0 \text { at } k_{s} \rightarrow \infty
$$

In other words, Phillips asymptotic means that energy is preserved and there is no leakage of energy to small scales. This point is in contradiction with the Kolmogorov picture of weak turbulence. We would like to make clear that the Phillips asymptotic $\omega^{-5}$ never can be obtained as the solution of the Hasselmann's equation.

Anyway, experimentalists systematically observe $\omega^{-5}$ tails in spectra of gravity waves, both in laboratory and in the ocean $[13,26]$. On our opinion, these tails appear in the conditions when local steepness is close to critical and the kinetic Hasselmann's equation in this case is not applicable, because the level of nonlinearity is very high.

Our slogan is

"Hasselmann equation and $\omega^{-5}$ spectrum are incompatible things". 


\section{Numerical simulation}

Numerical integration of kinetic equation for gravity waves on deep water (Hasselmann equation) was the subject of considerable efforts for last three decades. The "ultimate goal" of the effort—creation of the operational wave model for wave forecast based on direct solution of the Hasselmann equation-happened to be an extremely difficult computational problem due to mathematical complexity of the $S_{\mathrm{nl}}$ term, which requires calculation of a three-dimensional integral at every advance in time.

Historically, numerical methods of integration of kinetic equation for gravity waves exist in two "flavors". The first one is associated with works of Hasselmann et al. [16], Dungey and Hui [17], Masuda [18,19], Lavrenov [20], Polnikov [21] and is based on transformation of 6-fold into 3-fold integrals using $\delta$-functions . Such transformation leads to the appearance of integrable singularities, which creates additional difficulties in calculations of the $S_{\mathrm{nl}}$ term.

All numerical experiments show that the angle-averaged $S_{\mathrm{nl}}$ is a "two-lobe" function and consequently support the Kolmogorov scenario of wave turbulence. In some experiments [18,19,21] the Kolmogorov asymptotic $\omega^{-4}$ was observed.

The second type of models developed in works of Webb [22] and Resio and Perrie [23] uses direct calculation of resonant quadruplet contribution into $S_{\mathrm{nl}}$ integral based on the following property: given two fixed vectors $\vec{k}, \vec{k}_{1}$, another two $\vec{k}_{2}, \vec{k}_{3}$ are uniquely defined by the point "moving" along the resonant curve-locus.

Numerical simulation in the current work was performed with the help of modified version of the second type algorithm. Calculations were made on grid $71 \times 36$ points in the frequency-angle domain $[\omega, \theta]$ with exponential distribution of points in the frequency domain and uniform distribution of points in the angle direction.

We performed two series of experiments. In the first one we put in Eq. (2.4) an "artificial" driving and damping, which provide relatively broad "window of transparency". We assumed that damping is isotropic while instability can be either isotropic or anisotropic. These experiments are purely "academic". Their results cannot be applied to physical oceanography directly. They are designed to examine applicability of the weak turbulent theory and to validate a fundamental importance of weak turbulent Kolmogorov spectra. In these experiments we measure the approximate value of the first and the second Kolmogorov constants. Second series of experiments is modeling of the realistic case where Eq. (2.4) is supplied with wind-driven instability.

All cases of simulation started from uniformly distributed low level noise. Having in mind an application to real wind-driven sea waves we calibrate time of evolution in hours. The criterion for stop of the calculations was reaching of stationary or asymptotic regimes. Simulation was performed on Compaq Presario 1700 notebook computer featuring 850 Pentium III CPU with $256 \mathrm{MB}$ of RAM. Typical time of calculations varied between several dozen hours and several days.

\subsection{Isotropic case}

In the isotropic case

$$
\gamma(\omega, \theta)= \begin{cases}D_{1} \exp \left(-\left(\frac{\omega-\omega_{0}}{0.19}\right)^{4}\right) & \text { if } 0.63<\omega<1.26 \\ -D_{2}(\omega-0.63)^{2} & \text { if } \omega<0.63 \\ -D_{3}(\omega-5.65)^{2} & \text { if } \omega>5.65\end{cases}
$$

where $D_{i}, i=1,2,3$ are positive constants. Coefficient $D_{1}$ at (5.1) is defined from the condition of the smallness of the growth rate with respect to the corresponding local frequency. Negative components of (5.1) are high and low 


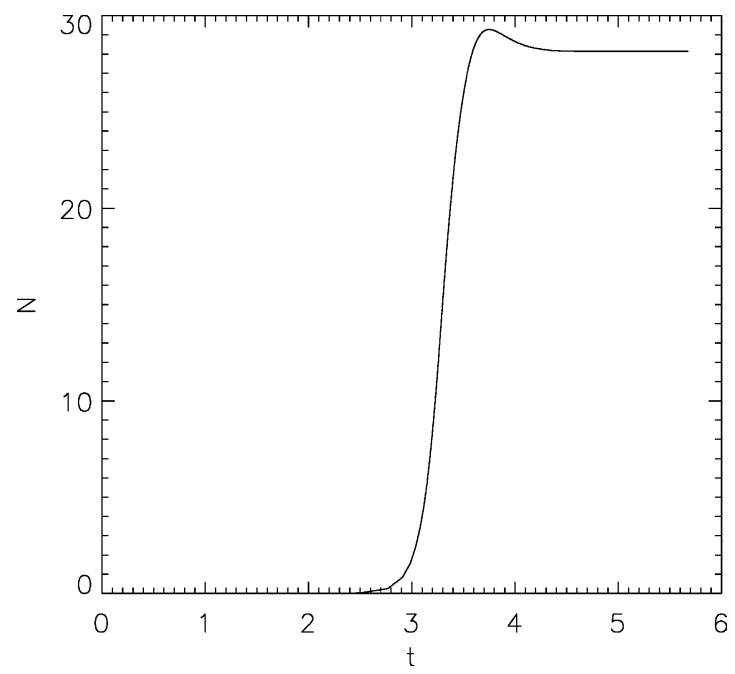

Fig. 1. Total wave action $N\left(\mathrm{~m}^{2} \mathrm{~s}\right)$ as the function of time $t(\mathrm{~h})$.

frequency damping terms, the only purpose of which is to absorb direct (energy) and inverse (wave action) cascades. Constants $D_{2}$ and $D_{3}$ as well as the frequencies $\omega=0.63$ and 5.65 are defined experimentally from the conditions of the effectiveness of the fluxes absorption and maximization of the inertial (forcing/damping rates free) interval with respect to $\omega$.

Fig. 1 shows evolution of wave action as a functions of time. The picture indicates that there are three main stages associated with system evolution: instability development, saturation at $t=3.7 \mathrm{~h}$, and final evolution into the stationary state. Energy demonstrates similar behavior.

Fig. 2 shows logarithm of energy distribution against logarithm of frequency at different moments of time. One can see formation of $\omega^{-4}$ asymptotic at finite moment of time. We interpret this fact as a vigorous support of the weak turbulent theory. We should stress that $\omega^{-4}$ asymptotic is very robust. Actually it appears in all our experiments.

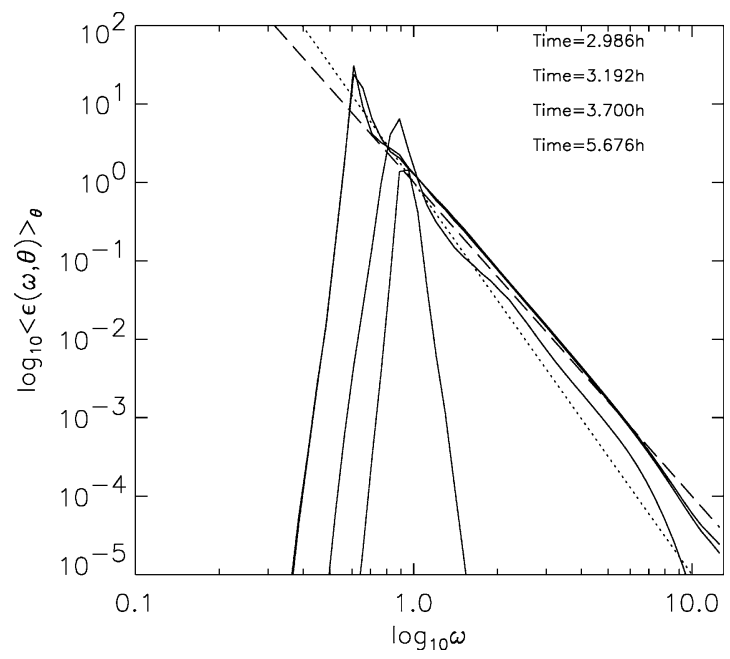

Fig. 2. $\log _{10}\langle\varepsilon(\omega, \theta)\rangle_{\theta}$ versus $\log _{10} \theta$ for different moments of time. Dotted line: function proportional to $\omega^{-5}$, dashed line: function proportional to $\omega^{-4}$ 


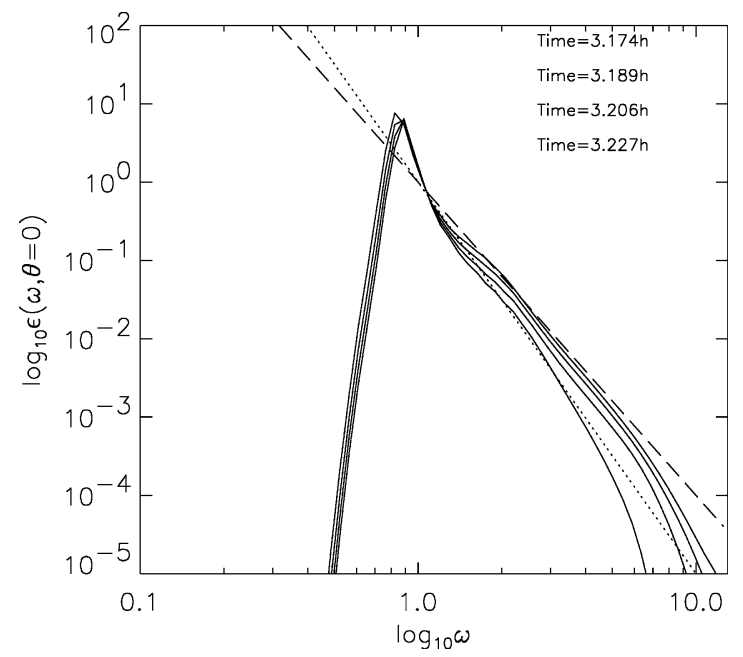

Fig. 3. Dynamics of the "shock" propagation for different equidistant moments of time: $\log _{10} \varepsilon(\omega, \theta=0)$ versus $\log _{10} \omega$. Dotted line: function proportional to $\omega^{-5}$, dashed line: function proportional to $\omega^{-4}$.

According to the predictions of Section 4, the $\omega^{-4}$ asymptotics develops in the explosive way. Energy spectra taken in four moments of time, close to the moment of explosion are shown in Fig. 3.

Next two figures display Kolmogorov flux of energy as a function of time measured in two different ways, by formula (4.1) (see Fig. 4) and (4.6) (see Fig. 5).

On the first stage energy grows exponentially until the "shock wave" in $k$-space reaches the Kolmogorov asymptotic. Then dissipation in high wave numbers explodes and the level of energy falls and reaches its stationary asymptotic value.

Fig. 6 presents the function

$$
\frac{\omega^{4}}{2 \pi P^{1 / 3} g^{4 / 3}} \int_{0}^{2 \pi} \varepsilon(\omega, \theta) \mathrm{d} \theta,
$$

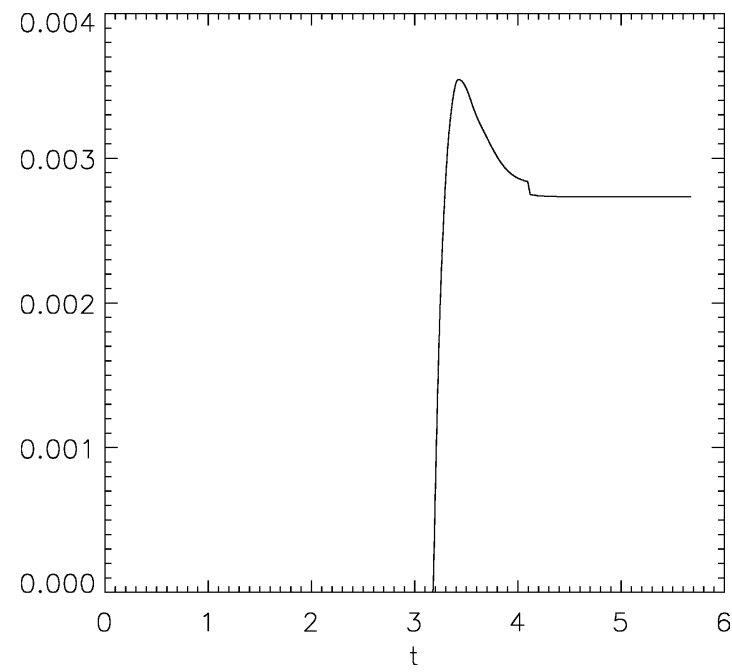

Fig. 4. Energy absorption $\left(\mathrm{m}^{2} / \mathrm{s}\right)$ as a function of time $t(\mathrm{~h})$. 


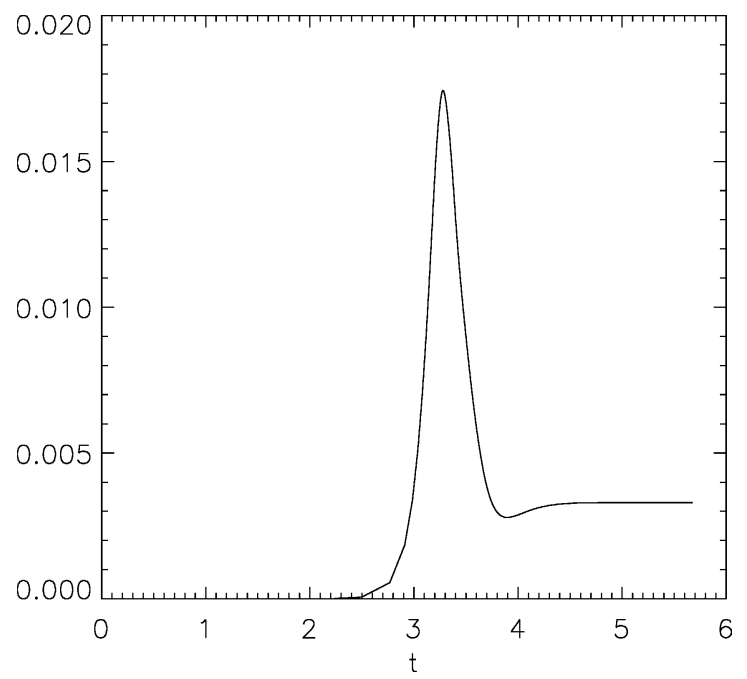

Fig. 5. Energy flux $\left(\mathrm{m}^{2} / \mathrm{s}\right)$ as a function of time $t(\mathrm{~h})$.

which gives for the first Kolmogorov constant

$$
0.35<C_{0}<0.45
$$

Fig. 6 shows that in the stationary state the spectrum has two different components- the Kolmogorov tail $\omega^{-4}$ and the sharp peak concentrated near the frequency $\omega \simeq 0.6$, corresponding to the lower edge of the instability region. Similar coexistence of "peak" and "tail" components is typical for wind-driven wave spectra, observed in the real ocean. In the standard JONSWAP spectrum a special parameter determining peakedness is provided (see also [9]).

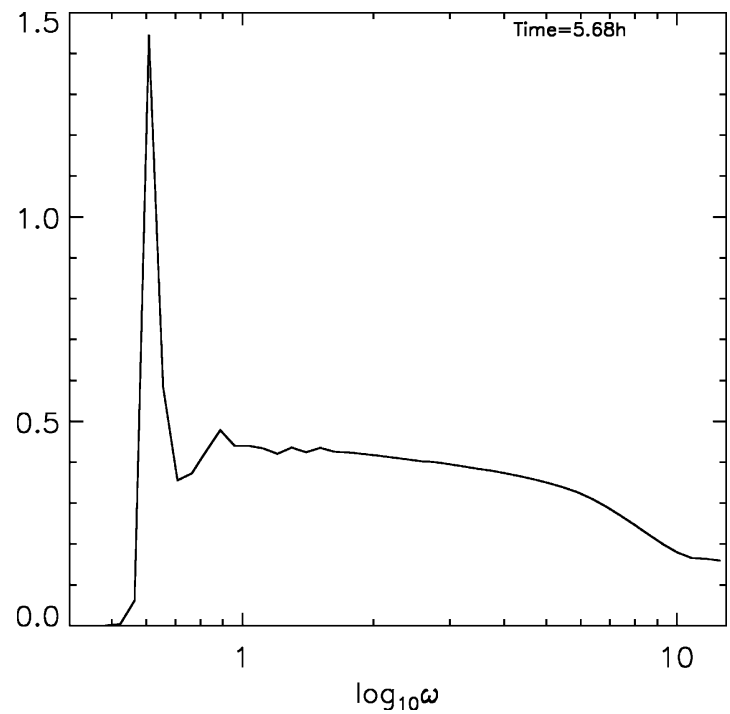

Fig. 6. Dimensionless function $\left(\omega^{4} / 2 \pi P^{1 / 3} g^{4 / 3}\right) \int_{0}^{2 \pi} \varepsilon(\omega, \theta) \mathrm{d} \theta$ as a function of $\log _{10} \omega$. 


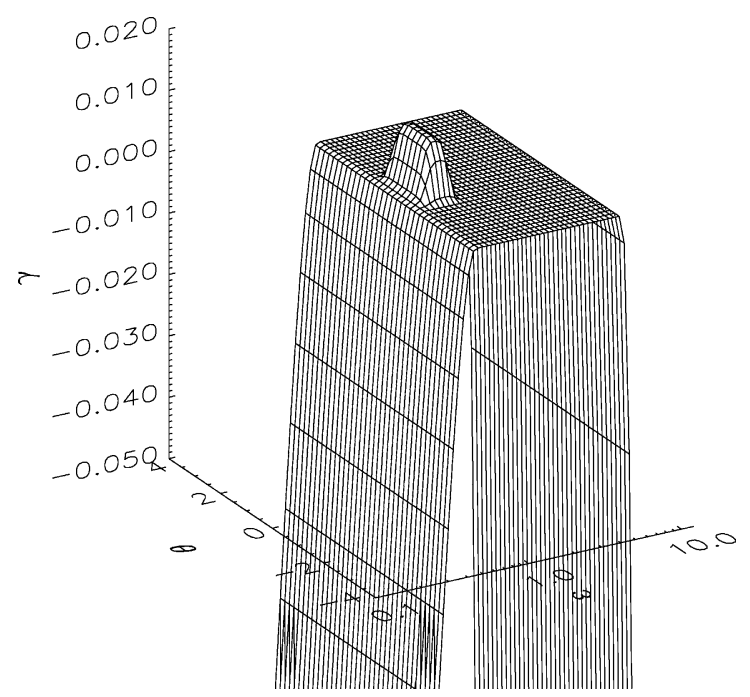

Fig. 7. Linear growth rate $\gamma\left(\mathrm{s}^{-1}\right)$ as the function of frequency $\omega(\mathrm{rad} / \mathrm{s})$ and angle $\theta(\mathrm{rad})$.

\subsection{Anisotropic case}

In the anisotropic case

$$
\gamma(\omega, \theta)= \begin{cases}D_{1} \exp \left(-\left(\frac{\omega-\omega_{0}}{0.19}\right)^{4}-\left(\frac{\theta}{\pi / 4}\right)^{8}\right) & \text { if } 0.63<\omega<1.26, \\ -D_{2}(\omega-0.63)^{2} & \text { if } \omega<0.63, \\ -D_{3}(\omega-5.65)^{2} & \text { if } \omega>5.65,\end{cases}
$$

where $D_{i}, i=1,2,3$ are positive constants, selected similarly to isotropic case. Fig. 7 shows distribution of damping and instability defined by (5.4).

This numerical simulation was motivated by the following reasons:

1. We want to be assured that weak turbulent Kolmogorov spectra are realized not only in the isotropic case. We would like to be completely sure that they play the same key role for essentially anisotropic spectra as well.

2. We planned to check once more the value of the first Kolmogorov constant $C_{0}$ and be sure that it is the same as in the isotropic case.

3. We want to trace the difference between the angle-averaged spectrum and its slice at $\theta=0$. We want also to find the value of the second Kolmogorov constant $C_{1}$.

The experiment shows that the stationary state is established similarly to the isotropic case. Typical saturation time for given forcing and damping is $t \simeq 0.68 \mathrm{~h}$.

Figs. 8-10 display line-levels of energy distribution at different moments of time. One can see that the stationary picture is bimodal and has double spike. A similar double spike picture is typical for experimental results [24,25].

Fig. 11 demonstrates a set of angle-averaged energy distribution taken in different moments of time. They are very close to a $\omega^{-4}$ law. Fig. 12 presents one-dimensional slices at $\theta=0$ for the energy distribution at the same times. Fig. 13 presents ratio of one-dimensional slices of spectra to angle-averaged spectra. One can see that one-dimensional spectra decay at $\omega \rightarrow \infty$ faster than the averaged energy spectrum, in accordance with Banner's observations [1]. We cannot identify, however, one-dimensional spectra with Phillips spectrum $\omega^{-5}$. Their decay is more slow and not uniform in $\omega$. 


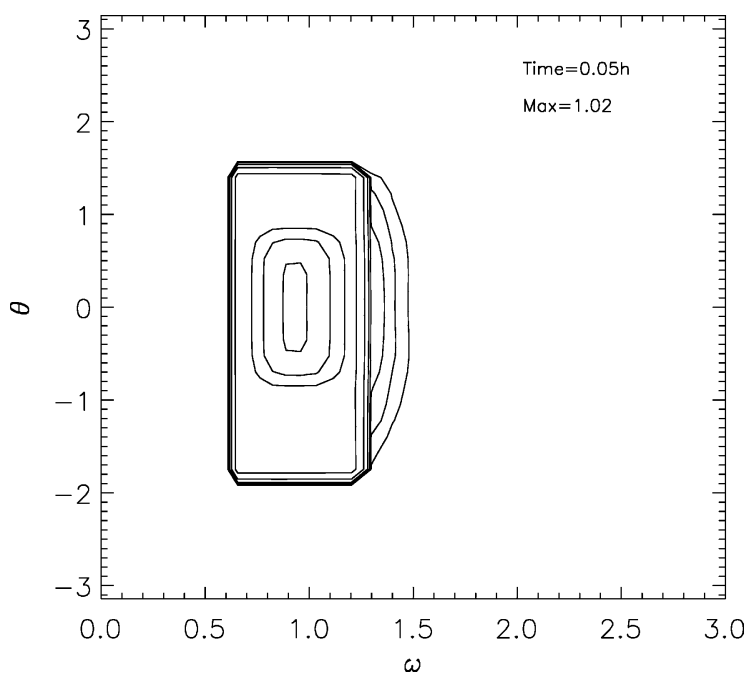

Fig. 8. Levels of constant energy density $\varepsilon(\omega, \theta)\left(\mathrm{m}^{2} \mathrm{~s}\right)$ versus $\omega(\mathrm{rad} / \mathrm{s})$ and angle $\theta(\mathrm{rad})$. Levels positioned as Max $/ 2^{n-1}$, where Max is the maximum of the distribution and $n=1, \ldots, 10$ are contour number starting from the highest contour.

In the anisotropic case we also saw explosive formation of spectra tails, similar to the isotropic case. Fig. 14 shows the energy spectrum development at four close equidistant time moments. As in the isotropic case one can notice that Kolmogorov spectrum establishment is strongly non-uniformly in space and time and looks like the "shock" propagation, in accordance with (4.16).

Fig. 15 shows the function $\left(\omega^{4} / P^{1 / 3}\right) \int_{0}^{2 \pi} \varepsilon \cos \theta d \theta$. If the formula (3.39) is correct, this plot should be proportional to $\omega^{-1}$. One can see that the correspondence is quite good.

Fig. 16 presents the function

$$
\frac{\omega^{4}}{2 \pi P^{1 / 3} g^{4 / 3}} \int_{0}^{2 \pi} \varepsilon(\omega, \theta) \mathrm{d} \theta,
$$

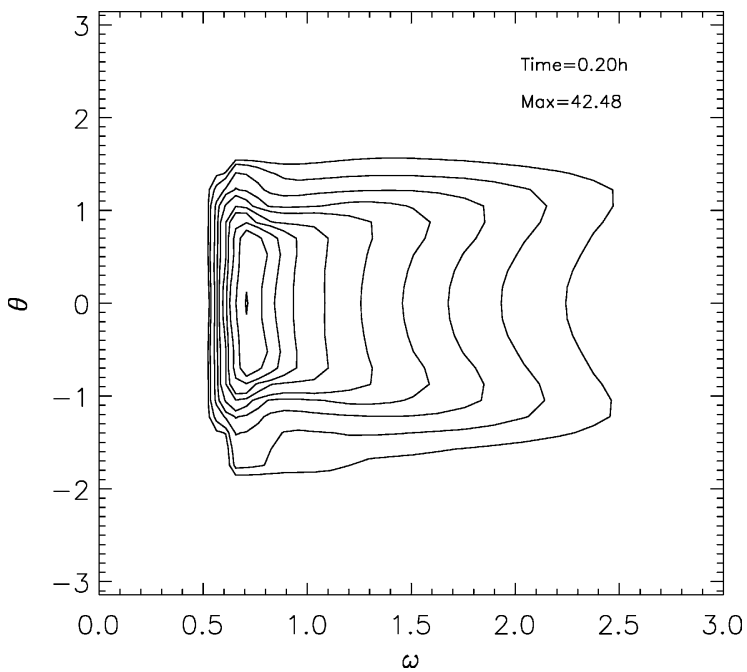

Fig. 9. Same as Fig. 8 for different times. 


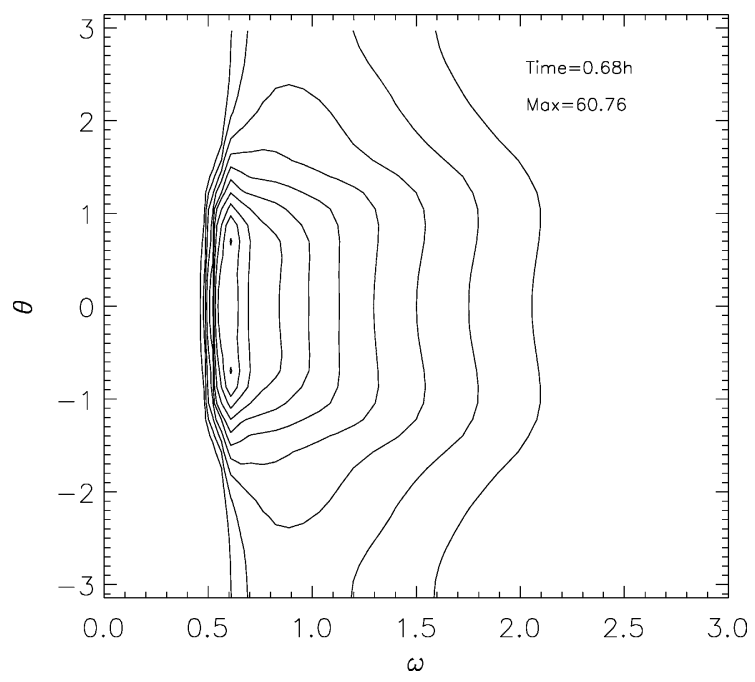

Fig. 10. Same as Fig. 8 for different times.

which gives the value of the first Kolmogorov constant in the anisotropic case

$$
0.33<C_{0}<0.37 \text {. }
$$

Fig. 17 presents the function

$$
\frac{P^{2 / 3} \omega^{5}}{\pi M g^{7 / 3}} \int_{0}^{2 \pi} \varepsilon(\omega, \theta) \cos \theta \mathrm{d} \theta,
$$

which gives the value of the second Kolmogorov constant

$$
0.18<C_{1}<0.27 \text {. }
$$

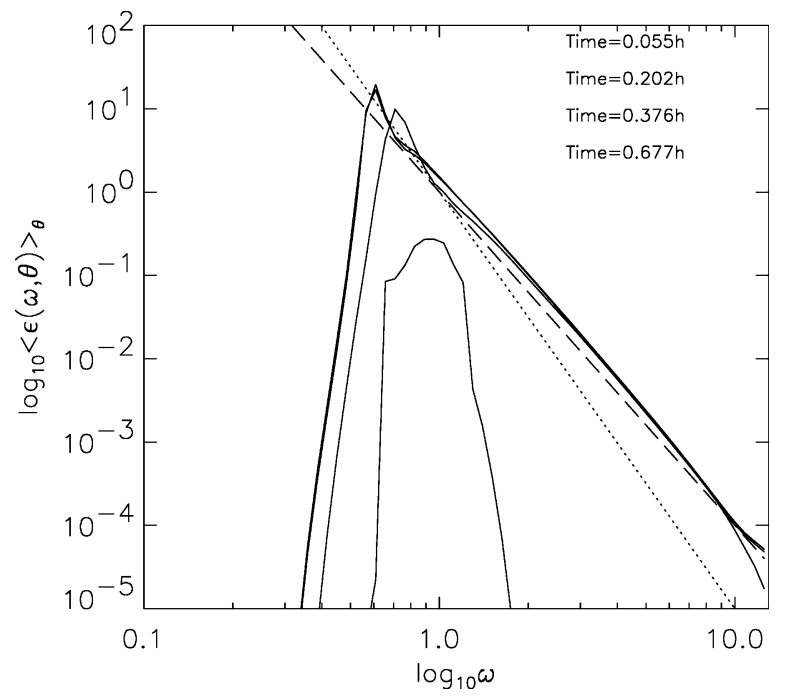

Fig. 11. $\log _{10}\langle\varepsilon(\omega, \theta)\rangle_{\theta}$ versus $\log _{10} \theta$ for different moments of time. Dotted line: function proportional to $\omega^{-5}$, dashed line: function proportional to $\omega^{-4}$. 


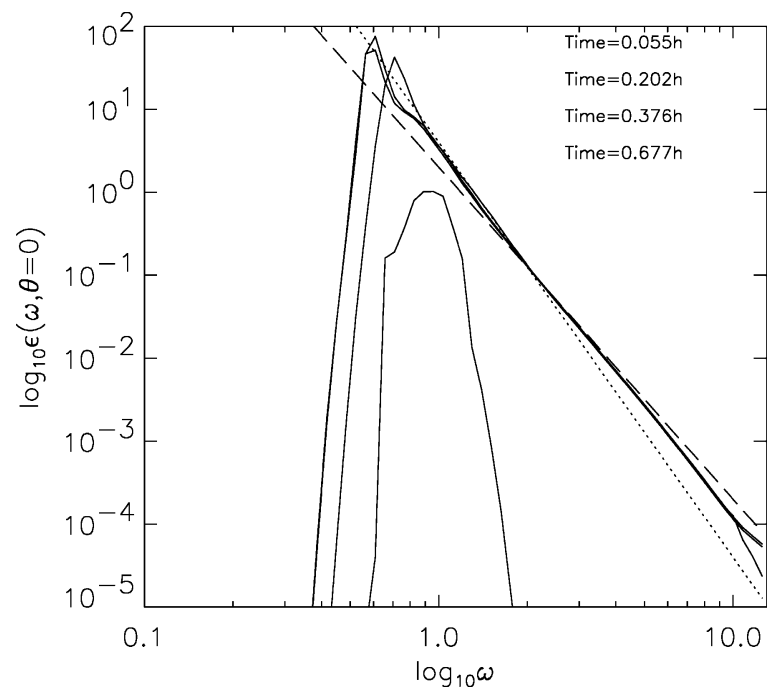

Fig. 12. $\log _{10} \varepsilon(\omega, \theta=0)$ versus $\log _{10} \theta$ for different moments of time. Dotted line: function proportional to $\omega^{-5}$, dashed line: function proportional to $\omega^{-4}$.

Figs. 16 and 17 show that in the anisotropic case we have again a combination of the spectral peak and the Kolmogorov-type tail.

\subsection{Wind forcing case}

In this section we present the results of modeling of the situation which is close to reality in maximum degree. We studied the surface waves excited by the wind in the angle-frequency domain $0<\theta<2 \pi$ and $\omega_{\min }<\omega<\omega_{\max }$,

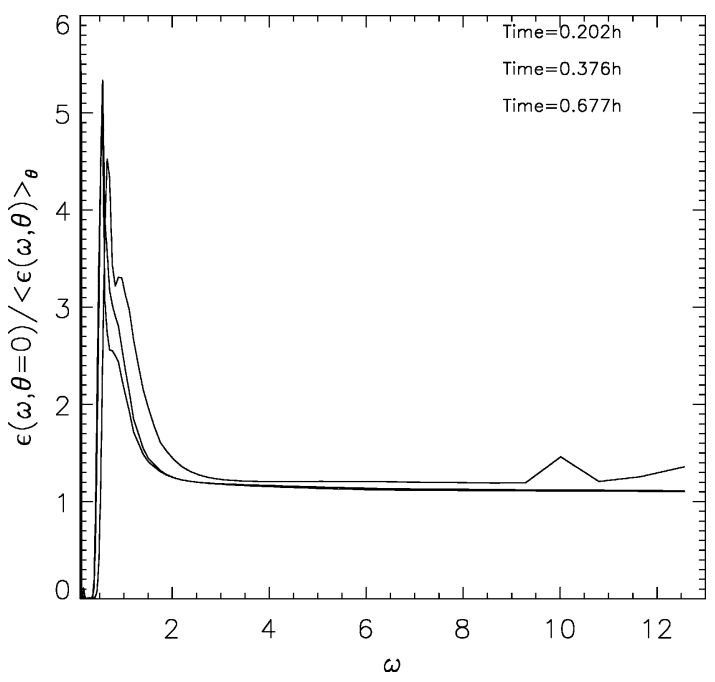

Fig. 13. Dimensionless ratio of one-dimensional slice $\varepsilon(\omega, \theta=0)$ to angle-averaged wave energy density $\langle\varepsilon(\omega, \theta)\rangle_{\theta} \operatorname{versus} \omega(\operatorname{rad} / \mathrm{s})$ for different moments of time. 


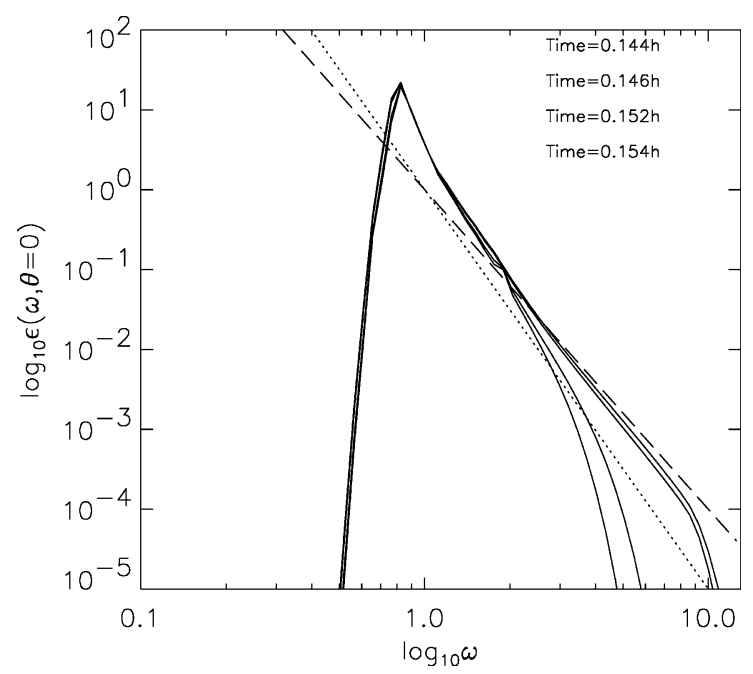

Fig. 14. Dynamics of the "shock" propagation for different equidistant moments of time: $\log _{10} \varepsilon(\omega, \theta=0)$ versus $\log _{10} \omega$. Dotted line: function proportional to $\omega^{-5}$, dashed line: function proportional to $\omega^{-4}$.

where $\omega_{\min }=0.06$ and $\omega_{\max }=12.56$. Initial conditions is the noise in energy space $\varepsilon_{\omega}=4 \times 10^{-5}$. Wind forcing and sink of energy at large $\omega$ are defined in accordance with (2.8) as

$$
\gamma(\omega, \theta)= \begin{cases}2 \times 10^{-4}\left(\frac{\omega}{\omega_{0}}-1\right)^{2} \omega \cos \theta & \text { if } \cos \theta>0 \text { and } \omega_{0}<\omega<\omega_{1}, \\ -D\left(\omega-\omega_{1}\right)^{2} & \text { if } \omega_{1}<\omega<\omega_{\max } \\ 0 & \text { otherwise }\end{cases}
$$

where $\omega_{0}=0.94$ (corresponds to wind velocity $U \simeq 10.4 \mathrm{~m} / \mathrm{s}$ ) and $\omega_{1}=8.48$. High-frequency damping is used to simulate infinite-capacity phase volume at high wave numbers. Constant $D$ and frequency $\omega_{1}$ are defined

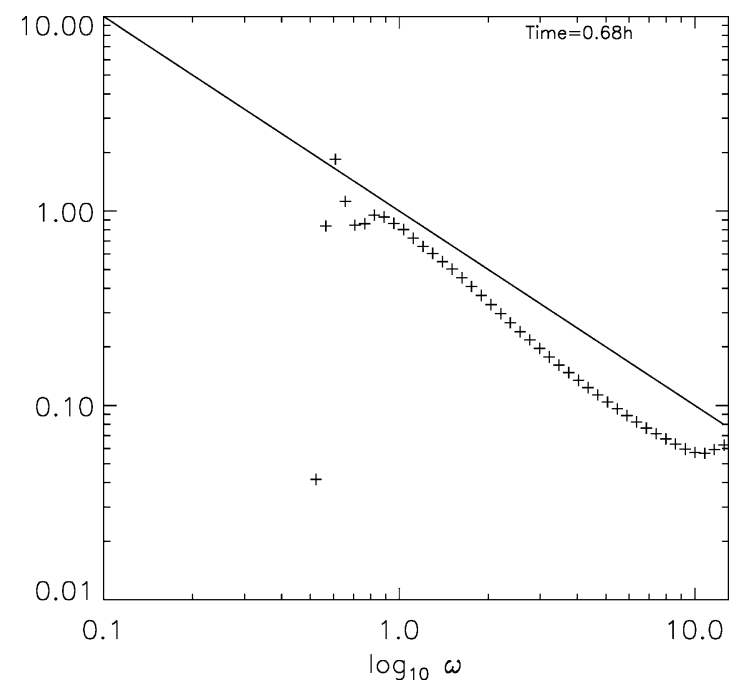

Fig. 15. Function $\log _{10}\left(\left(\omega^{4} / P^{1 / 3}\right) \int_{0}^{2 \pi} \cos \theta \mathrm{d} \theta\right)$ versus $\log _{10} \omega$ (crosses). Solid line: function, proportional to $\omega^{-1}$. 


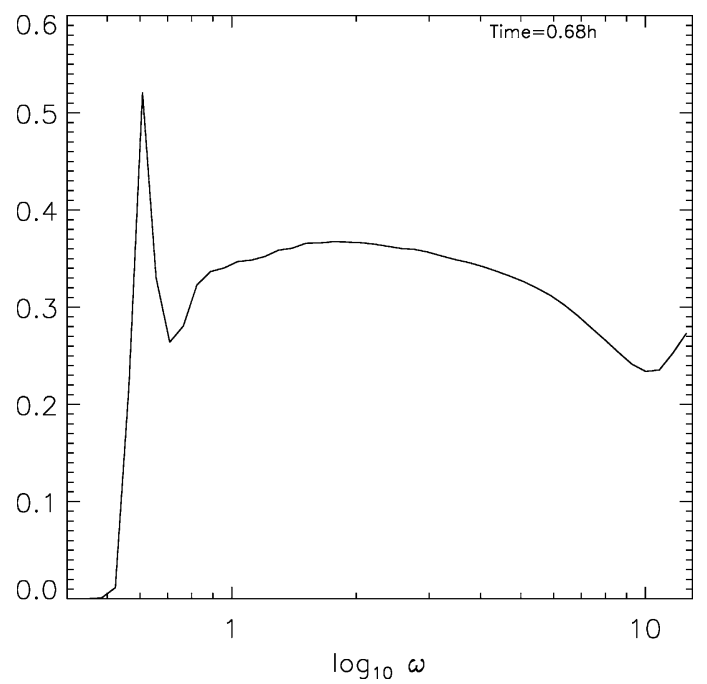

Fig. 16. Dimensionless function $\left(\omega^{4} / 2 \pi P^{1 / 3} g^{4 / 3}\right) \int_{0}^{2 \pi} \varepsilon(\omega, \theta) \mathrm{d} \theta$ versus $\log _{10} \omega$.

experimentally from the condition of the effectiveness of the energy flux absorption at high frequencies. As in reality, we did not provide any damping at small wave numbers. Fig. 18 shows distribution of damping and instability defined by (5.9).

We started our calculation from low-level noise and stopped them, when sea was close to its "mature state". As far as we know, nobody has performed similar experiments before.

The main purpose of our experiments is to prove that the weak turbulent four-wave interaction of gravity waves is a powerful enough mechanism to stabilize the wind-driven instability at relatively low level $k a \simeq 0.1-0.2$ and to provide fast enough down-shift of the peak of spectral density. This viewpoint is far from being widely accepted. Some authors consider that the random phase four-wave interaction is too weak a process to explain the rate of

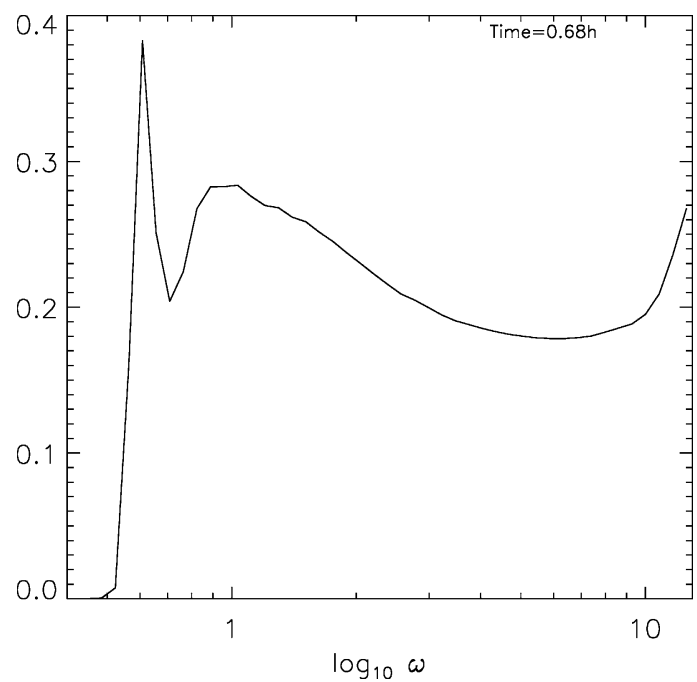

Fig. 17. Dimensionless function $\left(P^{2 / 3} \omega^{5} / \pi M g^{7 / 3}\right) \int_{0}^{2 \pi} \varepsilon(\omega, \theta) \cos \theta \mathrm{d} \theta$ versus $\log _{10} \omega$. 


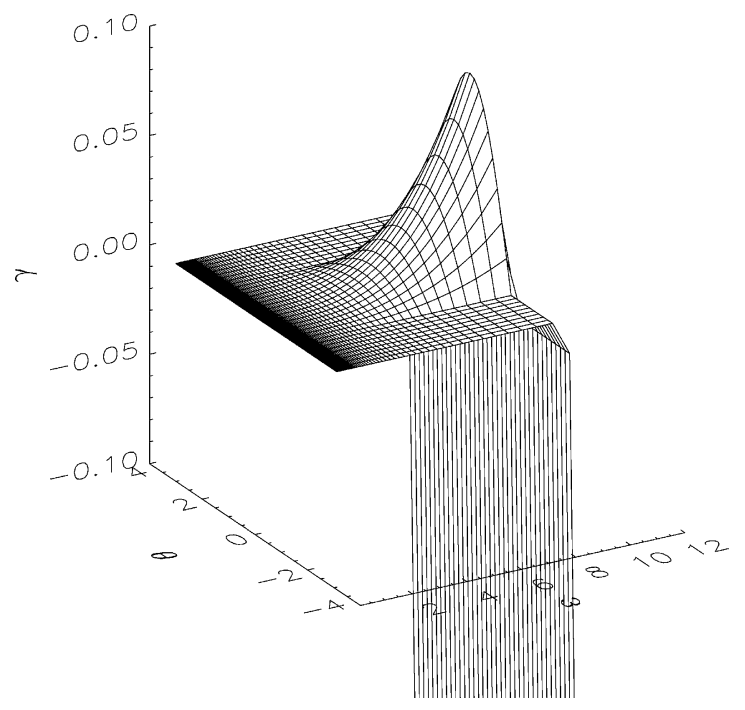

Fig. 18. Linear growth rate $\gamma\left(\mathrm{s}^{-1}\right)$ as the function of frequency $\omega(\mathrm{rad} / \mathrm{s})$ and angle $\theta(\mathrm{rad})$.

spectral evolution observed in the real ocean. Many authors traditionally believe that stationary spectra could appear only as a result of saturation of the instability by wave-breaking.

To argue with these points, we deliberately did not include the effects of wave-breaking in our consideration. We will show that the income of energy and momentum from wind is mostly compensated by Kolmogorov fluxes of these constants of motion. Income of wave action cannot be stabilized, thus the whole process is non-stationary. But at large times all spectral growth is concentrated in very small wave numbers, while at finite wave numbers it reaches a quasi-stationary state, which slowly changes in time. We should stress that at the current stage of our work we cannot perform detailed comparison of our theory with experiments, because in the most real cases spectra are non-uniform in space. They depend essentially on "fetch" (distance from the shore) and are "fetch-limited". Experimental data, pertaining to the spatially uniform ocean ("duration limited fetch") are scarce and not quite accurate. Some of this data are reviewed in the recent monograph of Young [41].

We performed comparison of our results with this data and found quite good coincidence. Anyway, we plan to perform the full-scale comparison of our numerical results with field and laboratory experiments as soon as we will have in our possession a numerical algorithm for modeling of non-stationary limiting fetch situation. Then we will consider more carefully the possible role of wave-breaking in balance of energy in the wind-driven sea.

In our experiments the wind velocity was $10.4 \mathrm{~m} / \mathrm{s}$. The total duration of simulation was about $4 \mathrm{~h}$ of physical time. We discuss below the results of these experiments.

First of all, one can see that four-wave interaction is a very powerful and fast mechanism of the instability saturation. Fig. 19 presents total wave action as a function of time. After few minutes of exponential growth, described by linear theory, wave action stabilizes and turns into a linear function of time.

Total energy $H$ and significant wave height $a_{\mathrm{s}}$ defined by the standard formula

$$
a_{\mathrm{s}}=4 \sqrt{H}
$$

grow more slowly (Figs. 20 and 21):

$$
H(t) \simeq t^{0.79},
$$




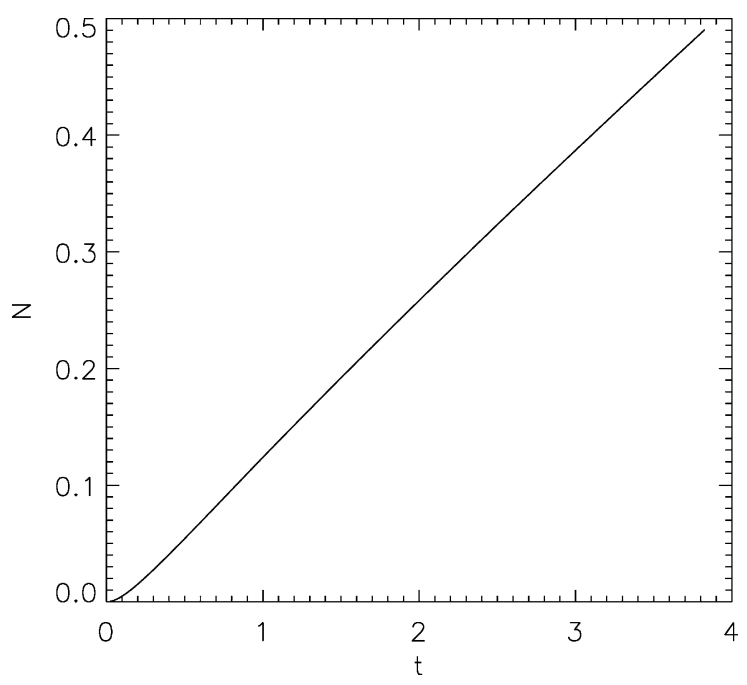

Fig. 19. Total wave action $N\left(\mathrm{~m}^{2} \mathrm{~s}\right)$ as the function of time $t(\mathrm{~h})$.

$$
a_{\mathrm{s}}(t) \simeq t^{0.39} .
$$

At the end of the experiment significant wave height reaches the value $a_{\mathrm{s}} \simeq 3 \mathrm{~m}$.

Four-wave interaction provides an efficient down-shift. Average frequency $\langle\omega\rangle$ decays approximately as

$$
\langle\omega\rangle \simeq t^{-0.27},
$$

and reaches the value $\langle\omega\rangle \simeq 1.2 \mathrm{rad} / \mathrm{s}$, see Fig. 22 .

Dependence of the average slope on time $\mu=\langle k a\rangle$ is presented in Fig. 23. Here $a=\sqrt{2 H}$ is a characteristic amplitude of the wave. One can see that in the initial stage of evolution $a$ reaches its maximum value $\mu=0.27$

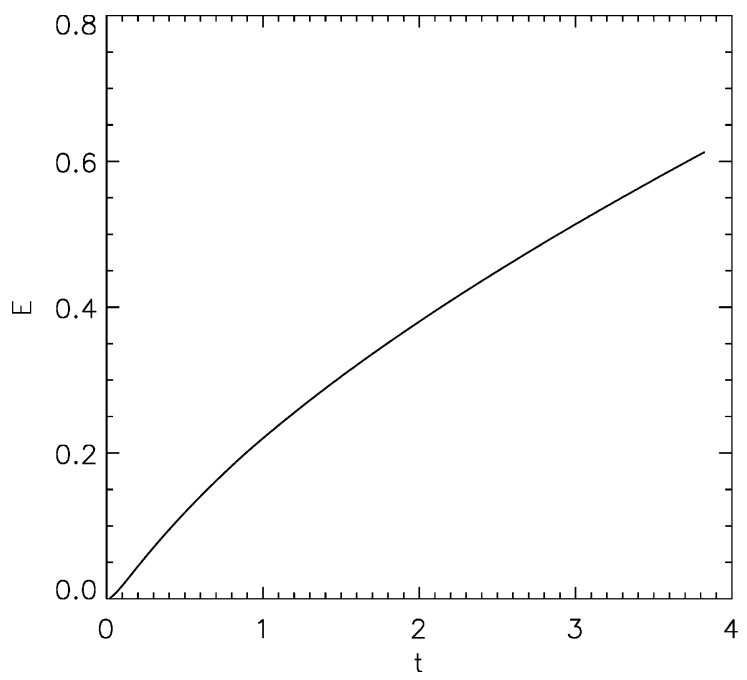

Fig. 20. Total wave energy $E\left(\mathrm{~m}^{2}\right)$ as the function of time $t(\mathrm{~h})$. 


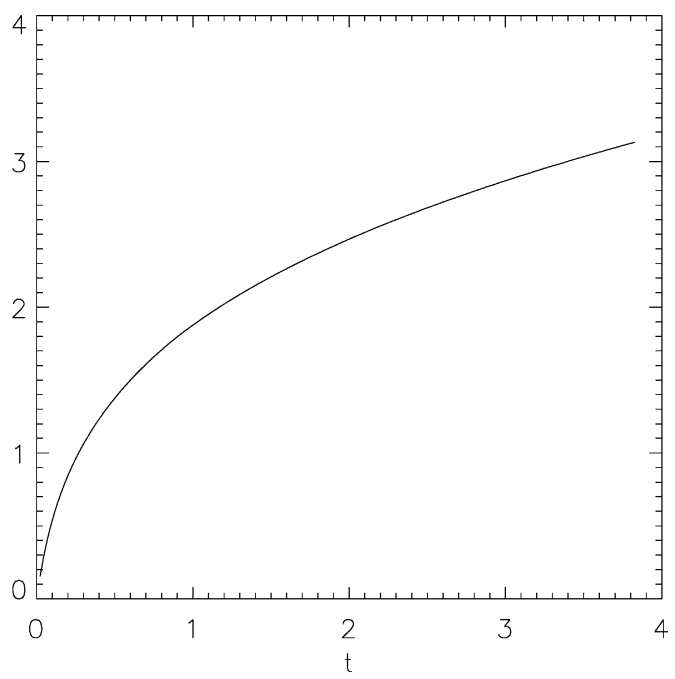

Fig. 21. Significant wave height $\left\langle a_{\mathrm{s}}\right\rangle(\mathrm{m})$ as the function of time $t(\mathrm{~h})$.

and decreases slowly to $\mu=0.15$. Figs. 24 and 25 demonstrate comparison of our calculations with experimental results presented in the book of Young [41].

One should stress that $4 \mathrm{~h}$ of physical time of numerical experiment is a moderate time-frame, and even at the end of our calculations the waves are relatively young. Recently we performed longer calculations and can pre-announce some new results. After $10 \mathrm{~h}$ of physical time, the average frequency $\langle\omega\rangle$ down-shifts to $0.6 \mathrm{rad} / \mathrm{s}$, while slope decreases down to $\mu \simeq 0.1$, in accordance with estimates obtained from analysis of experimental data.

Fig. 26 presents level-lines of the spectral density at the end of calculations. The spectral peak is narrow in angle and is concentrated inside the range $\langle\delta \theta\rangle<30^{\circ}$. The spectral tail is broader. Fig. 27 presents evolution of averaged spectra in logarithmic scale. It is clear that spectral tail is close to $\omega^{-4}$. In Fig. 28 "compensated" spectra

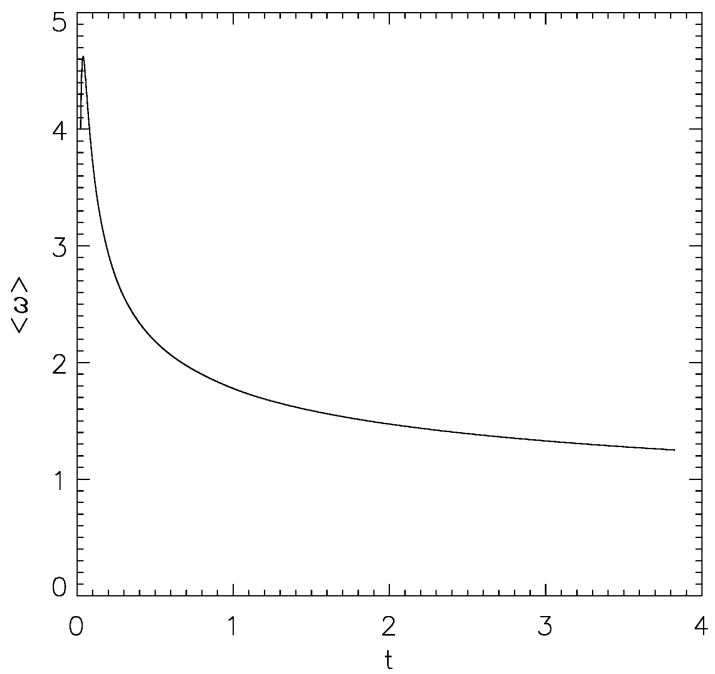

Fig. 22. Average frequency $\langle\omega\rangle(\mathrm{rad} / \mathrm{s})$ as the function of time $t(\mathrm{~h})$. 


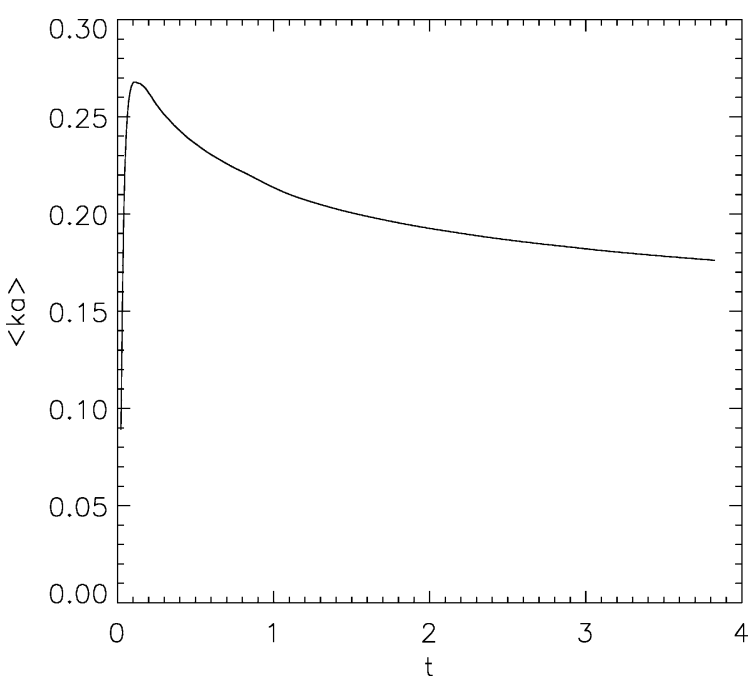

Fig. 23. Dimensionless average wave slope $\langle k a\rangle$ as the function of time $t(\mathrm{~h})$.

$\omega^{4} \varepsilon_{\omega}$ are plotted in natural scale. Fig. 29 presents one-dimensional slices of wave energy in different moments of time. Fig. 30 presents the ratio of one-dimensional spectra to averaged spectrum in natural scale. One can see that one-dimensional spectra decay faster than average in accordance with Banner's observation.

Figs. 31-33 demonstrate different terms in the equation

$$
\frac{\partial n}{\partial t}=S_{\mathrm{nl}}+\gamma_{k} n_{k}
$$

for three time moments. One can see that in the area of spectral maximum $\partial n / \partial t$ is almost equal to $S_{\mathrm{nl}}$ and forcing terms are small even in the initial stage of the process. On the contrary, in the area of spectral tail $\partial n / \partial t$ is very small, and the instability term $\gamma_{k} n_{k}$ is compensated by the nonlinear interaction term $S_{\mathrm{nl}}$. In this case the spectrum

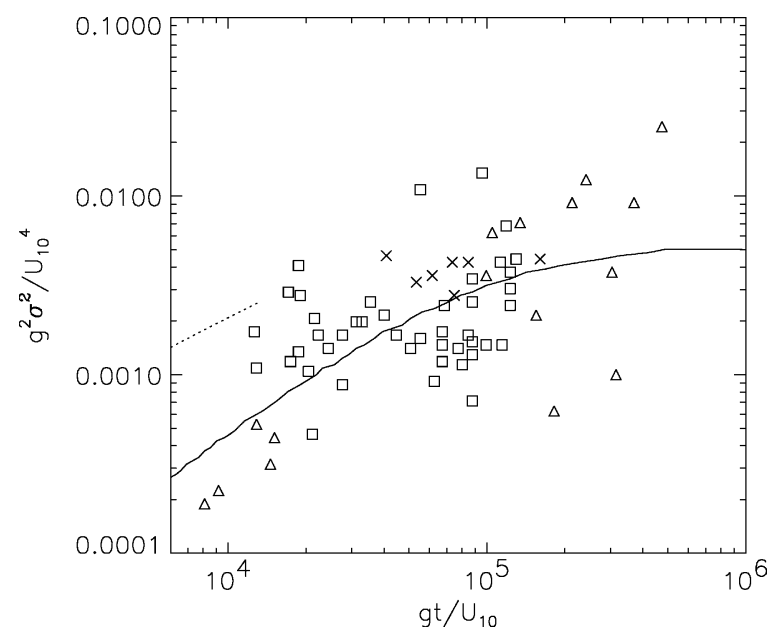

Fig. 24. Data compiled by Wiegel (1961) showing duration limited growth of dimensionless energy $g^{2} \sigma^{2} / U_{10}^{4}$ versus dimensionless duration $g t / U_{10}$. The solid line is data fit by CERC. Data taken from [41]. Dotted line is data from current numerical experiment. 


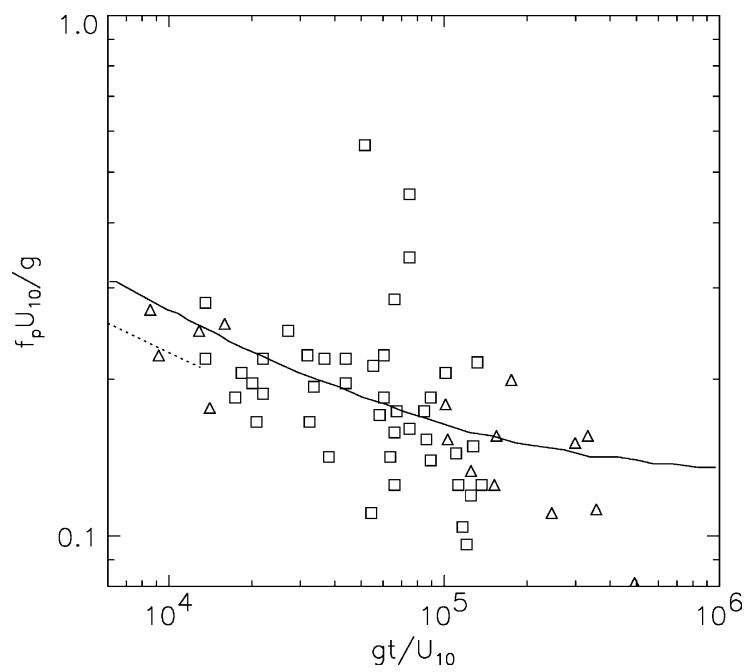

Fig. 25. Data compiled by Wiegel (1961) showing duration limited growth of non-dimensional frequency $f_{p} U_{10} / g$ versus non-dimensional duration $g t / U_{10}$. The solid line is data fit by CERC. Data taken from [41]. Dotted line is data from current numerical experiment.

is quasi-stationary. These figures clearly demonstrate that $S_{\mathrm{nl}}$ alone arrests the growth of instability on the very low level. To make this fact more conspicuous, we present the same picture in natural scales in Figs. 34-36, performing a zoom on the vertical axis.

Figs. 37-39 present integrated action, energy and momentum inputs

$$
Q(\Omega)=\frac{2}{g^{2}} \int_{0}^{\Omega} \int_{0}^{2 \pi} \gamma n \omega^{3} \mathrm{~d} \omega \mathrm{d} \theta
$$

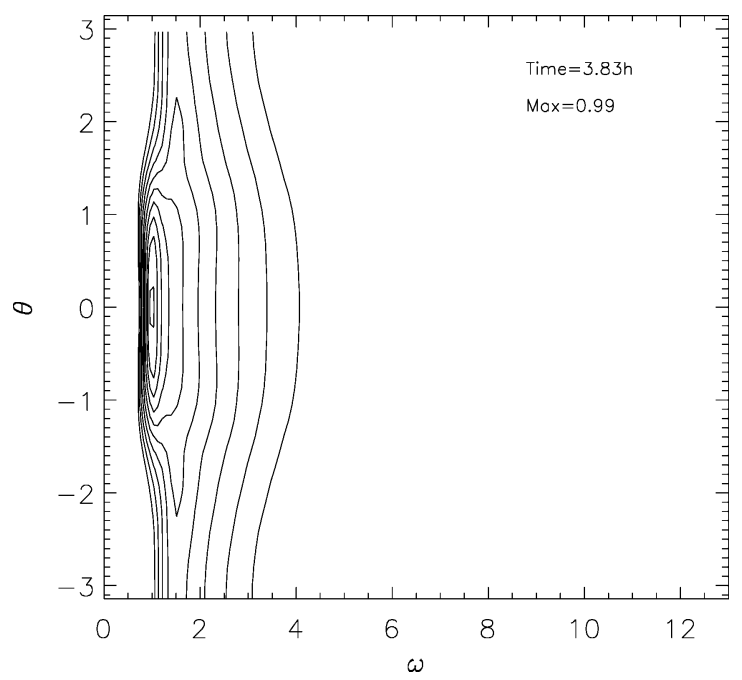

Fig. 26. Levels of constant energy density $\varepsilon(\omega, \theta)\left(\mathrm{m}^{2} \mathrm{~s}\right)$ versus $\omega(\mathrm{rad} / \mathrm{s})$ and angle $\theta(\mathrm{rad})$. Levels positioned as Max $/ 2^{n-1}$, where Max is the maximum of the distribution and $n=1, \ldots, 10$ are contour number starting from the highest contour. 


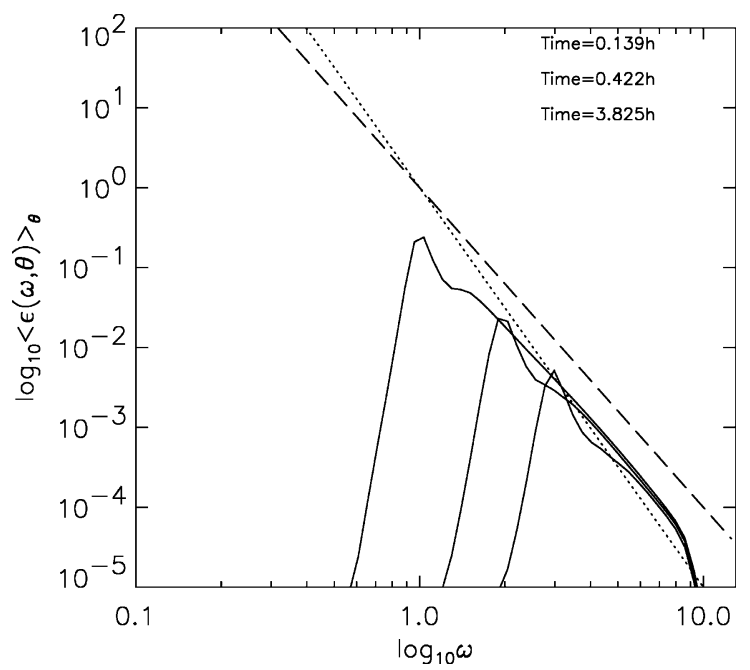

Fig. 27. $\log _{10}\langle\varepsilon(\omega, \theta)\rangle_{\theta}$ versus $\log _{10} \theta$ for different moments of time. Dotted line: function proportional to $\omega^{-5}$, dashed line: function proportional to $\omega^{-4}$.

$$
\begin{aligned}
& P(\Omega)=\frac{2}{g^{2}} \int_{0}^{\Omega} \int_{0}^{2 \pi} \gamma n \omega^{4} \mathrm{~d} \omega \mathrm{d} \theta \\
& M(\Omega)=\frac{2}{g^{3}} \int_{0}^{\Omega} \int_{0}^{2 \pi} \gamma n \omega^{5} \cos \theta \mathrm{d} \omega \mathrm{d} \theta
\end{aligned}
$$

as the functions of frequency $\Omega$.

All these three functions reach their maximum values at the end of the range of instability. Thus $Q_{\max }, P_{\max }, M_{\max }$ are total income of motion constants from the wind per unit of time. Apparently, $Q_{\infty}, P_{\infty}, M_{\infty}$ taken at the end of

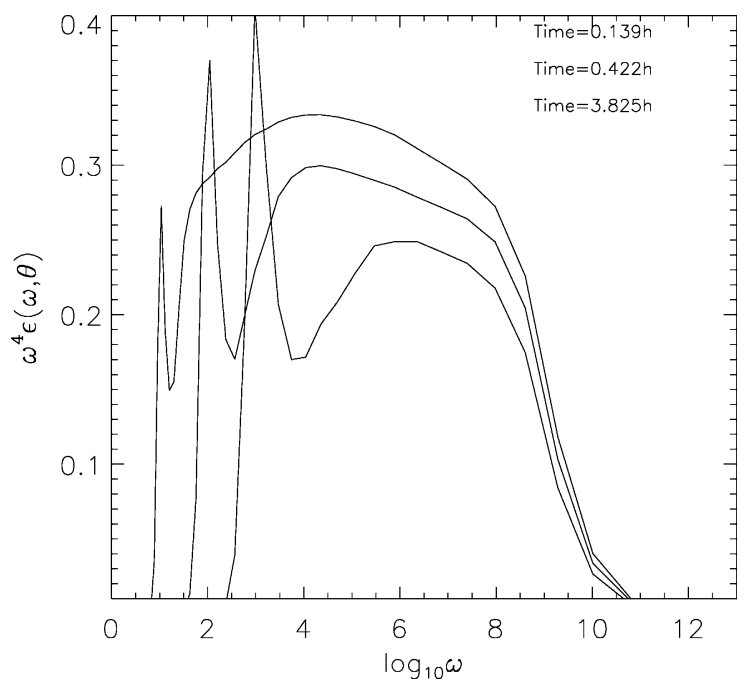

Fig. 28. "Compensated" spectra $\omega^{4} \varepsilon_{\omega}\left(\mathrm{m}^{2} / \mathrm{s}^{3}\right)$ as a function of $\omega(\mathrm{rad} / \mathrm{s})$ for different times. 


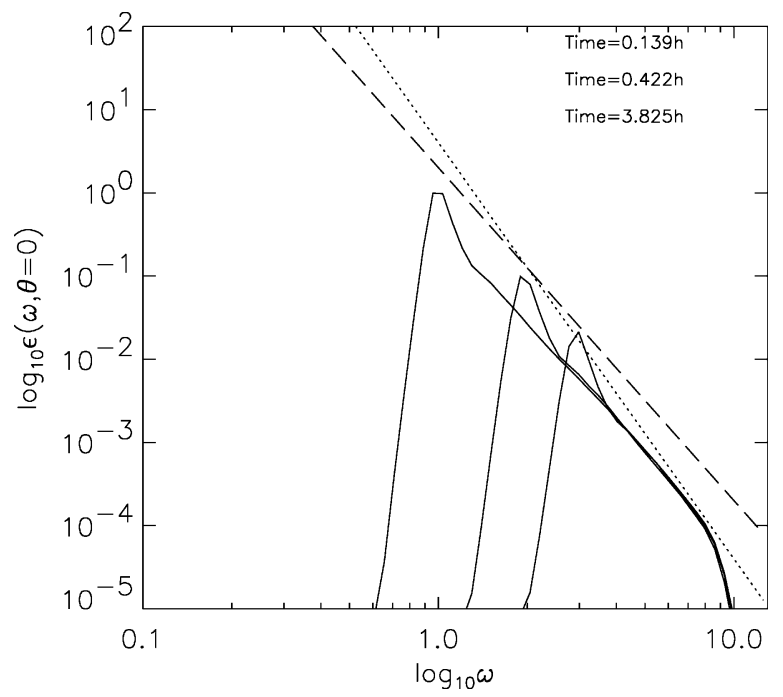

Fig. 29. $\log _{10} \varepsilon(\omega, \theta=0)$ versus $\log _{10} \theta$ for different moments of time. Dotted line: function proportional to $\omega^{-5}$, dashed line: function proportional to $\omega^{-4}$.

damping region can be identified with time derivatives of total action, energy and momentum. One can see that at the end of calculation

$$
\begin{aligned}
\frac{Q_{\infty}}{Q_{\max }} & \simeq 0.80, \\
\frac{P_{\infty}}{P_{\max }} & \simeq 0.45, \\
\frac{M_{\infty}}{M_{\max }} & \simeq 0.29 .
\end{aligned}
$$

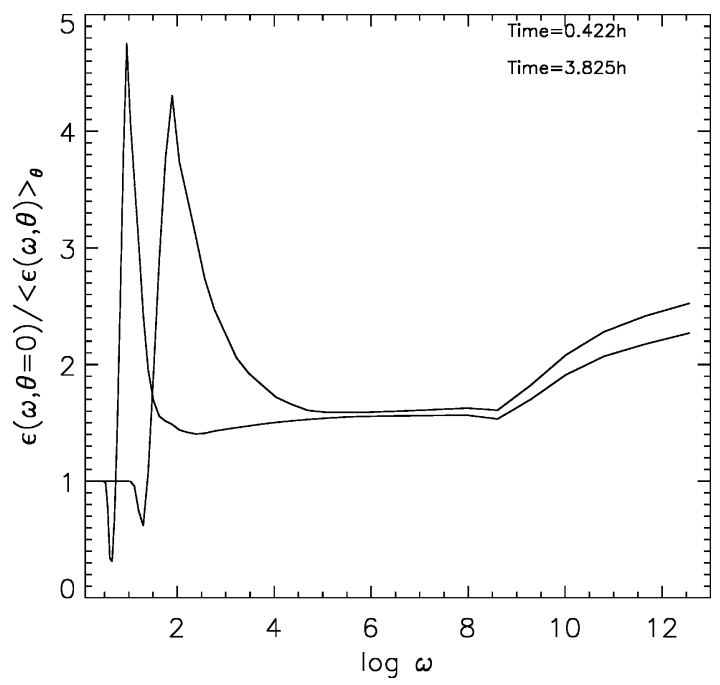

Fig. 30. Dimensionless ratio of one-dimensional slice $\varepsilon(\omega, \theta=0)$ to angle-averaged wave energy density $\langle\varepsilon(\omega, \theta)\rangle_{\theta} \operatorname{versus} \omega(\mathrm{rad} / \mathrm{s})$ for different moments of time. 


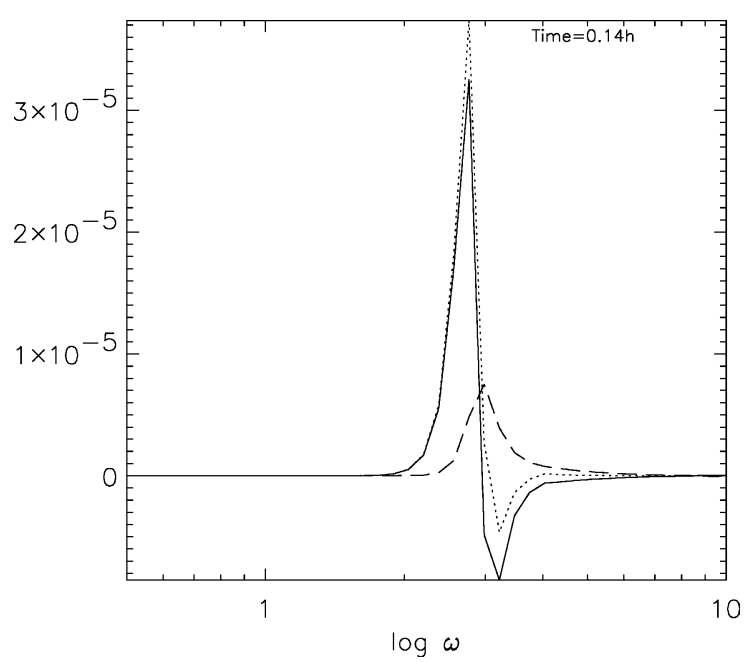

Fig. 31. Angle-averaged terms $\left(\mathrm{m}^{4}\right)$ in the kinetic equation $(\partial n / \partial t)=S_{\mathrm{nl}}+\gamma n$ as the function of $\log _{10} \omega$ for $t=0.14$ (h). Solid line: $\int_{0}^{2 \pi} S_{\mathrm{nl}} \mathrm{d} \theta$, dashed line: $\int_{0}^{2 \pi} \gamma n \mathrm{~d} \theta$, dotted line: $\int_{0}^{2 \pi}(\partial n / \partial t) \mathrm{d} \theta$.

Weak turbulent theory predicts that at $t \rightarrow \infty$ ratios $P_{\infty} / P_{\max } \rightarrow 0, M_{\infty} / M_{\max } \rightarrow 0$, while $Q_{\infty} / Q_{\max } \rightarrow \lambda<1$, where $\lambda$ is some constant.

In other words, for very developed sea waves almost all energy and momentum are transferred from air to sea and carried by Kolmogorov fluxes to high frequency region. Our calculation clearly demonstrates this tendency.

\section{Conclusions}

The method presented here for numerical solution of Hasselmann's kinetic equation for gravity waves makes it possible to solve this equation in a broad domain that covers more than two decades in frequency. This algorithm makes it possible to perform $10^{4}-10^{5}$ time steps without accumulating significant error or developing any instabilities. Results based on the numerical simulations conducted here support the theory of Kolmogorov spectra for weak turbulence not only in its basic points but also in many details. Some key conclusions from our investigation are as follows:

1. In accordance with weak turbulence theory, we found that energy and momentum of the wave ensemble are not preserved. Both of these quantities are "leaked" to the region of very small scales where it is assumed that they are lost due to local dissipative processes (wave breaking, generation of capillary waves, etc.). This leakage is an important part of the formation of the universal Kolmogorov spectrum.

2. Directional energy spectra in the equilibrium range are proportional to $\omega^{-4}$. This result is very persistent and in all numerical experiments, the $\omega^{-4}$ Kolmogorov spectrum appears in very early stages of the simulation and persists in both stationary and non-stationary stages of spectral development.

3. A very important aspect of the simulations conducted here was the development of a quasi-stationary wave spectrum under wind forcing, without the need for a dissipation mechanism in the spectral peak region. Previous investigations (for example [35,36]) have been unable to achieve this result and consequently concluded that wave breaking in the spectral peak region must be an important component in developing fully developed seas. Our results suggest that primary wave dissipation region is most likely located only in the high-frequency tail of the spectrum. 
4. Fluxes of momentum and energy through the equilibrium range (Kolmogorov region) of the wave spectrum are observed to produce a bimodal angular distribution of energy at high frequencies. This is consistent with observations of sea waves in nature [24].

It should be recognized here that our results are consistent with several previous empirical investigations. First of all, behavior of integral characteristics of wave ensemble (average energy and mean frequency) is in accordance with experimental data on limited duration observations. Laboratory data from the classic study of Toba [11] clearly showed that wave spectra at laboratory scales contain characteristic $\omega^{-4}$ equilibrium ranges, rather than the $\omega^{-5}$ form initially hypothesized by Phillips [37] and adopted in many early spectral parameterizations of ocean spectra [38,39]. More recent studies, including Mitsuyasu et al. [29], Forristall [26], Donelan et al. [9] and Battjes et al. [31] (see also $[27,28,30,32]$ ), have all shown that the equilibrium range in deep-water ocean waves follows an $\omega^{-4}$ form. Resio et al. [40] have shown that the infinite-depth form for the equilibrium form is $k^{-5 / 2}$, which is also consistent with the Kolmogorov spectrum and asymptotically approaches $\omega^{-4}$ form in deep water.

The findings here are quite robust and hopefully will be applied to the practical problems. Present wave prediction models are based on fairly crude parameterizations of the nonlinear energy transfers. In large part due to inaccuracies in these parameterizations, these models have had to include strong dissipation in the spectral peak region to inhibit wave growth as full development is approached. Possibly because of the dominance of the dissipative term in the energy balance near full development, these models consistently under-predict wave heights in larger storms. Results from this study could be used to reformulate the complete energy balance equation for wave generation, propagation and decay, which could lead to substantially improved predictions in the near future.

\section{Acknowledgements}

The research presented in this paper was conducted under the US Army Corps of Engineers, RDT\&E program, grant DACA 42-00-C0044, ONR grant N00014-98-1-0070 and NSF grant NDMS0072803. The Chief of Engineers has granted permission for publication. This support is gratefully acknowledged. The authors are grateful to I. Lavrenov for valuable comments.

\section{Appendix A}

Presented formulae are valid for any depth. They are taken from the paper [33]. Variables $\psi, \omega$ are canonical. They obey the Hamiltonian equations

$$
\frac{\partial \eta}{\partial t}=-\frac{\delta H}{\delta \Psi}, \quad \frac{\partial \Psi}{\partial t}=\frac{\delta H}{\delta \eta}
$$

where $H$ is the total energy of fluid. It is presented by the series in powers of characteristic slope $k a$ in terms of Fourier transforms:

$$
\begin{aligned}
& H=H_{0}+H_{1}+H_{2}+\cdots, \quad H_{0}=\frac{1}{2} \int\left[A_{k}\left|\Psi_{k}\right|^{2}+B_{k}\left|\eta_{k}\right|^{2}\right] \mathrm{d} k, \quad A_{k}=k \tanh (k h), \quad B_{k}=g, \\
& H_{1}=\frac{1}{2(2 \pi)} \int L^{(1)}\left(\vec{k}_{1}, \vec{k}_{2}\right) \Psi_{k_{1}} \Psi_{k_{2}} \delta\left(\vec{k}_{1}+\vec{k}_{2}+\vec{k}_{3}\right) \mathrm{d} k_{1} \mathrm{~d} k_{2} \mathrm{~d} k_{3}, \\
& H_{2}=\frac{1}{2(2 \pi)^{2}} \int L^{(2)}\left(\vec{k}_{1}, \vec{k}_{2}, \vec{k}_{3}, \vec{k}_{4}\right) \Psi_{k_{1}} \Psi_{k_{2}} \eta_{k_{3}} \eta_{k_{4}} \delta\left(\vec{k}_{1}+\vec{k}_{2}+\vec{k}_{3}+\vec{k}_{4}\right) \mathrm{d} k_{1} \mathrm{~d} k_{2} \mathrm{~d} k_{3} \mathrm{~d} k_{4},
\end{aligned}
$$


where

$L^{(1)}\left(\vec{k}_{1}, \vec{k}_{2}\right)=-\left(\vec{k}_{1} \vec{k}_{2}\right)-\left|k_{1}\right|\left|k_{2}\right| \tanh k_{1} h \tanh k_{2} h$

and

$$
\begin{aligned}
L^{(2)}\left(\vec{k}_{1}, \vec{k}_{2}, \vec{k}_{3}, \vec{k}_{4}\right)= & \frac{1}{4}\left|k_{1}\right|\left|k_{2}\right| \tanh k_{1} h \tanh k_{1} h\left[\frac{-2\left|k_{1}\right|}{\tanh k_{1} h}+\frac{-2\left|k_{2}\right|}{\tanh k_{2} h}+\left|\vec{k}_{1}+\vec{k}_{3}\right| \tanh \left|\vec{k}_{1}+\vec{k}_{3}\right| h\right. \\
& \left.+\left|\vec{k}_{2}+\vec{k}_{3}\right| \tanh \left|\vec{k}_{2}+\vec{k}_{3}\right| h+\left|\vec{k}_{1}+\vec{k}_{4}\right| \tanh \left|\vec{k}_{1}+\vec{k}_{4}\right| h+\left|\vec{k}_{2}+\vec{k}_{4}\right| \tanh \left|\vec{k}_{2}+\vec{k}_{4}\right| h\right] \\
= & \frac{1}{4} A_{1} A_{2}\left[-\frac{2 k_{1}^{2}}{A_{1}}-\frac{2 k_{2}^{2}}{A_{2}}+A_{1+3}+A_{2+3}+A_{1+4}+A_{2+4}\right] .
\end{aligned}
$$

Cubical terms in Hamiltonian are excluded by canonical transformation. The Hamiltonian is given by the infinite series

$$
a_{k}=a_{k}^{(0)}+a_{k}^{(1)}+a_{k}^{(2)}+\cdots
$$

where

$$
\begin{aligned}
& a_{k}^{(0)}=b_{k}, \\
& a_{k}^{(1)}=\int \Gamma^{(1)}\left(\vec{k}, \vec{k}_{1}, \vec{k}_{2}\right) b_{k_{1}} b_{k_{2}} \delta\left(\vec{k}-\vec{k}_{1}-\vec{k}_{2}\right) \mathrm{d} k_{1} \mathrm{~d} k_{2}-2 \int \Gamma^{(1)}\left(\vec{k}_{2}, \vec{k}, \vec{k}_{1}\right) b_{k_{1}}^{*} b_{k_{2}} \delta\left(\vec{k}+\vec{k}_{1}-\vec{k}_{2}\right) \mathrm{d} k_{1} \mathrm{~d} k_{2} \\
& +\int \Gamma^{(2)}\left(\vec{k}, \vec{k}_{1}, \vec{k}_{2}\right) b_{k_{1}}^{*} b_{k_{2}}^{*} \delta\left(\vec{k}+\vec{k}_{1}+\vec{k}_{2}\right) \mathrm{d} k_{1} \mathrm{~d} k_{2} \\
& a_{k}^{(2)}=\int B\left(\vec{k}, \vec{k}_{1}, \vec{k}_{2}, \vec{k}_{3}\right) b_{k_{1}}^{*} b_{k_{2}} b_{k_{3}} \delta\left(\vec{k}-\vec{k}_{1}-\vec{k}_{2}-\vec{k}_{3}\right) \mathrm{d} k_{1} \mathrm{~d} k_{2} \mathrm{~d} k_{3}+\cdots, \\
& \Gamma^{(1)}\left(\vec{k}, \vec{k}_{1}, \vec{k}_{2}\right)=-\frac{1}{2} \frac{V^{(1,2)}\left(\vec{k}, \vec{k}_{1}, \vec{k}_{2}\right)}{\omega_{k}-\omega_{k_{1}}-\omega_{k_{2}}}, \quad \Gamma^{(2)}\left(\vec{k}, \vec{k}_{1}, \vec{k}_{2}\right)=-\frac{1}{2} \frac{V^{(0,3)}\left(\vec{k}, \vec{k}_{1}, \vec{k}_{2}\right)}{\omega_{k}-\omega_{k_{1}}-\omega_{k_{2}}}, \\
& B\left(\vec{k}, \vec{k}_{1}, \vec{k}_{2}, \vec{k}_{3}\right)=\Gamma^{(1)}\left(\vec{k}_{1}, \vec{k}_{2}, \vec{k}_{1}-\vec{k}_{2}\right) \Gamma^{(1)}\left(\vec{k}_{3}, \vec{k}, \vec{k}_{3}-\vec{k}\right)+\Gamma^{(1)}\left(\vec{k}_{1}, \vec{k}_{3}, \vec{k}-\vec{k}_{3}\right) \Gamma^{(1)}\left(\vec{k}_{2}, \vec{k}, \vec{k}_{2}-\vec{k}\right) \\
& -\Gamma^{(1)}\left(\vec{k}, \vec{k}_{2}, \vec{k}-\vec{k}_{2}\right) \Gamma^{(1)}\left(\vec{k}_{3}, \vec{k}_{1}, \vec{k}_{3}-\vec{k}_{1}\right)-\Gamma^{(1)}\left(\vec{k}_{1}, \vec{k}_{3}, \vec{k}_{1}-\vec{k}_{3}\right) \Gamma^{(1)}\left(\vec{k}_{2}, \vec{k}_{1}, \vec{k}_{2}-\vec{k}_{1}\right) \\
& -\Gamma^{(1)}\left(\vec{k}+\vec{k}_{1}, \vec{k}, \vec{k}_{1}\right) \Gamma^{(1)}\left(\vec{k}_{2}+\vec{k}_{3}, \vec{k}, \vec{k}_{1}\right)+\Gamma^{(2)}\left(-\vec{k}-\vec{k}_{1}, \vec{k}, \vec{k}_{1}\right) \Gamma^{(2)}\left(-\vec{k}_{2}-\vec{k}_{3}, \vec{k}_{2}, \vec{k}_{3}\right) \text {. }
\end{aligned}
$$

Then

$$
\begin{aligned}
n_{k}= & N_{k}-\frac{g}{2} \int \frac{\left|V^{(1,2)}\left(\vec{k}, \vec{k}_{1}, \vec{k}_{2}\right)\right|^{2}}{\left(\omega_{k}-\omega_{k_{1}}-\omega_{k_{2}}\right)^{2}}\left(N_{k_{1}} N_{k_{2}}-N_{k} N_{k_{1}}-N_{k} N_{k_{2}}\right) \delta\left(\vec{k}-\vec{k}_{1}-\vec{k}_{2}\right) \mathrm{d} k_{1} \mathrm{~d} k_{2} \\
& -\frac{g}{2} \int \frac{\left|V^{(1,2)}\left(\vec{k}, \vec{k}_{1}, \vec{k}_{2}\right)\right|^{2}}{\left(\omega_{k_{1}}-\omega_{k}-\omega_{k_{2}}\right)^{2}}\left(N_{k_{1}} N_{k_{2}}+N_{k} N_{k_{1}}-N_{k} N_{k_{2}}\right) \delta\left(\vec{k}_{1}-\vec{k}-\vec{k}_{2}\right) \mathrm{d} k_{1} \mathrm{~d} k_{2} \\
& -\frac{g}{2} \int \frac{\left|V^{(1,2)}\left(\vec{k}_{2}, \vec{k}, \vec{k}_{1}\right)\right|^{2}}{\left(\omega_{k_{2}}-\omega_{k}+\omega_{k_{1}}\right)^{2}}\left(N_{k_{1}} N_{k_{2}}+N_{k} N_{k_{2}}-N_{k} N_{k_{1}}\right) \delta\left(\vec{k}_{2}-\vec{k}-\vec{k}_{1}\right) \mathrm{d} k_{1} \mathrm{~d} k_{2} \\
& -\frac{g}{2} \int \frac{\left|V^{(0,3)}\left(\vec{k}, \vec{k}_{1}, \vec{k}_{2}\right)\right|^{2}}{\left(\omega_{k}+\omega_{k_{1}}+\omega_{k_{2}}\right)^{2}}\left(N_{k_{1}} N_{k_{2}}+N_{k} N_{k_{1}}+N_{k} N_{k_{2}}\right) \delta\left(\vec{k}-\vec{k}_{1}-\vec{k}_{2}\right) \mathrm{d} k_{1} \mathrm{~d} k_{2},
\end{aligned}
$$


where

$$
\begin{aligned}
& V^{(1,2)}\left(\vec{k}, \vec{k}_{1}, \vec{k}_{2}\right)=\frac{1}{4 \pi \sqrt{2}}\left\{\left(\frac{A_{k} B_{k_{1}} C_{k_{2}}}{B_{k} A_{k_{1}} A_{k_{2}}}\right)^{1 / 4} L^{(1)}\left(\vec{k}_{1}, \vec{k}_{2}\right)-\left(\frac{B_{k} A_{k_{1}} B_{k_{2}}}{A_{k} A_{k_{1}} A_{k_{2}}}\right)^{1 / 4} L^{(1)}\left(-\vec{k}, \vec{k}_{1}\right)\right. \\
& \left.-\left(\frac{B_{k} B_{k_{1}} A_{k_{2}}}{A_{k} A_{k_{1}} B_{k_{2}}}\right)^{1 / 4} L^{(1)}\left(-\vec{k}, \vec{k}_{2}\right)\right\}, \\
& V^{(0,3)}\left(\vec{k}, \vec{k}_{1}, \vec{k}_{2}\right)=\frac{1}{4 \pi \sqrt{2}}\left\{\left(\frac{A_{k} B_{k_{1}} B_{k_{2}}}{B_{k} A_{k_{1}} A_{k_{2}}}\right)^{1 / 4} L^{(1)}\left(\vec{k}_{1}, \vec{k}_{2}\right)+\left(\frac{B_{k} A_{k_{1}} B_{k_{2}}}{A_{k} B_{k_{1}} A_{k_{2}}}\right)^{1 / 4} L^{(1)}\left(\vec{k}, \vec{k}_{1}\right)\right. \\
& \left.+\left(\frac{B_{k} B_{k_{1}} A_{k_{2}}}{A_{k} A_{k_{1}} B_{k_{2}}}\right)^{1 / 4} L^{(1)}\left(\vec{k}, \vec{k}_{2}\right)\right\} \text {. }
\end{aligned}
$$

\section{Appendix B}

The coefficient of four-wave interaction for pure gravity waves on deep water was calculated by many authors since Hasselmann (1962). We present here relatively compact expression for this coefficient (see [33]):

$$
\begin{aligned}
T_{1234}= & \frac{1}{2}\left(\tilde{T}_{1234}+\tilde{T}_{2134}\right), \\
\tilde{T}_{1234}= & -\frac{1}{16 \pi^{2}} \frac{1}{\left(k_{1} k_{2} k_{3} k_{4}\right)^{1 / 4}} \\
& \times\left\{\begin{array}{l}
- \\
-12 k_{1} k_{2} k_{3} k_{4}-2\left(\omega_{1}+\omega_{2}\right)^{2}\left[\omega_{3} \omega_{4}\left(\left(\vec{k}_{1} \cdot \vec{k}_{2}\right)-k_{1} k_{2}\right)+\omega_{1} \omega_{2}\left(\left(\vec{k}_{3} \vec{k}_{4}\right)-k_{3} k_{4}\right)\right] \frac{1}{g^{2}}
\end{array}\right. \\
& -2\left(\omega_{1}-\omega_{3}\right)^{2}\left[\omega_{2} \omega_{4}\left(\left(\vec{k}_{1} \vec{k}_{3}\right)+k_{1} k_{3}\right)+\omega_{1} \omega_{3}\left(\left(\vec{k}_{2} \vec{k}_{4}\right)+k_{2} k_{4}\right)\right] \frac{1}{g^{2}} \\
& -2\left(\omega_{1}-\omega_{4}\right)^{2}\left[\omega_{2} \omega_{3}\left(\left(\vec{k}_{1} \vec{k}_{4}\right)+k_{1} k_{4}\right)+\omega_{1} \omega_{4}\left(\left(\vec{k}_{2} \vec{k}_{3}\right)+k_{2} k_{3}\right)\right] \frac{1}{g^{2}} \\
& +\left[\left(\vec{k}_{1} \cdot \vec{k}_{2}\right)+k_{1} k_{2}\right]\left[\left(\vec{k}_{3} \cdot \vec{k}_{4}\right)+k_{3} k_{4}\right]+\left[-\left(\vec{k}_{1} \cdot \vec{k}_{3}\right)+k_{1} k_{3}\right]\left[-\left(\vec{k}_{2} \cdot \vec{k}_{4}\right)+k_{2} k_{4}\right] \\
& +\left[-\left(\vec{k}_{1} \cdot \vec{k}_{4}\right)+k_{1} k_{4}\right]\left[-\left(\vec{k}_{2} \cdot \vec{k}_{3}\right)+k_{2} k_{3}\right]+4\left(\omega_{1}+\omega_{2}\right)^{2} \frac{\left[\left(\vec{k}_{1} \cdot \vec{k}_{2}\right)-k_{1} k_{2}\right]\left[\left(\vec{k}_{3} \cdot \vec{k}_{4}\right)-k_{3} k_{4}\right]}{\omega_{1+2}^{2}-\left(\omega_{1}+\omega_{2}\right)^{2}} \\
& +4\left(\omega_{1}-\omega_{3}\right)^{2} \frac{\left[\left(\vec{k}_{1} \cdot \vec{k}_{3}\right)+k_{1} k_{3}\right]\left[\left(\vec{k}_{2} \cdot \vec{k}_{4}\right)+k_{2} k_{4}\right]}{\omega_{1-3}^{2}-\left(\omega_{1}-\omega_{3}\right)^{2}} \\
& \left.+4\left(\omega_{1}-\omega_{4}\right)^{2} \frac{\left[\left(\vec{k}_{1} \cdot \vec{k}_{4}\right)+k_{1} k_{4}\right]\left[\left(\vec{k}_{2} \cdot \vec{k}_{3}\right)+k_{2} k_{3}\right]}{\omega_{1-4}^{2}-\left(\omega_{1}-\omega_{4}\right)^{2}}\right\} .
\end{aligned}
$$

Here $\omega_{i}=\sqrt{g\left|k_{i}\right|}$.

For coinciding wave vectors $T_{12,12}=T_{12}$ :

$$
\begin{aligned}
& T_{12}=-\frac{1}{8 \pi^{2}} \frac{1}{\left(k_{1} k_{2}\right)^{1 / 2}}\left\{3 k_{1}^{2} k_{2}^{2}+\left(\vec{k}_{1} \vec{k}_{2}\right)^{2}-4 \omega_{1} \omega_{2}\left(\vec{k}_{1} \cdot \vec{k}_{2}\right)\left(k_{1}+k_{2}\right) \frac{1}{g^{2}}+2 \frac{\left(\omega_{1}+\omega_{2}\right)^{2}\left[\vec{k}_{1} \cdot \vec{k}_{2}-k_{1} k_{2}\right]^{2}}{\omega_{1+2}^{2}-\left(\omega_{1}+\omega_{2}\right)^{2}}\right. \\
& \left.+2 \frac{\left(\omega_{1}-\omega_{2}\right)^{2}\left[\vec{k}_{1} \vec{k}_{2}+k_{1} k_{2}\right]^{2}}{\omega_{1-2}^{2}-\left(\omega_{1}-\omega_{2}\right)^{2}}\right\}
\end{aligned}
$$


In the one-dimensional case the formula (B.1) becomes remarkably simple (see [34]):

$$
T_{12}=\frac{1}{2 \pi^{2}} \begin{cases}k_{1}^{2} k_{2}, & k_{1}<k_{2}, \\ k_{1} k_{2}^{2}, & k_{1}>k_{2} .\end{cases}
$$

In a general case $T_{12}$ has the asymptotic

$$
T_{12} \simeq \frac{1}{2 \pi^{2}} k_{1} k_{2}^{2} \cos \theta
$$

at $k_{1} \gg k_{2}$.

\section{Appendix C}

To determine the equation, describing the general Kolmogorov solution (3.36) one defines the following function:

$$
\begin{aligned}
F(\omega, \theta)=4 \pi \int_{0}^{\infty} & \mathrm{d} \omega_{1} \int_{0}^{\omega_{3}} \mathrm{~d} \omega_{2} \int_{\omega}^{\infty} \mathrm{d} \omega_{3} \int_{0}^{2 \pi} \mathrm{d} \theta_{1} \int_{0}^{2 \pi} \mathrm{d} \theta_{2} \int_{0}^{2 \pi} \mathrm{d} \theta_{3} \delta\left(\omega+\omega_{1}-\omega_{2}-\omega_{3}\right) \\
& \times \delta\left(\omega \cos \theta+\omega_{1} \cos \theta_{1}-\omega_{2} \cos \theta_{2}-\omega_{3} \cos \theta_{3}\right) \delta\left(\omega \sin \theta+\omega_{1} \sin \theta_{1}-\omega_{2} \sin \theta_{2}-\omega_{3} \sin \theta_{3}\right) \\
& \times\left[\omega^{3} N_{\omega_{1}} N_{\omega_{2}} N_{\omega_{3}}+\omega_{1}^{3} N_{\omega} N_{\omega_{2}} N_{\omega_{3}}-\omega_{2}^{3} N_{\omega} N_{\omega_{1}} N_{\omega_{3}}-\omega_{3}^{3} N_{\omega} N_{\omega_{1}} N_{\omega_{2}}\right] \mid T_{\omega \omega_{1} \omega_{2} \omega_{3},\left.\theta \theta_{1} \theta_{2} \theta_{3}\right|^{2}}
\end{aligned}
$$

$$
N(\omega, \theta)=\frac{2 \omega^{3}}{g} n(\omega, \theta),
$$

$N(\omega, \theta) \mathrm{d} \omega \mathrm{d} \theta=n_{k} \mathrm{~d} k$,

and find its Fourier coefficients

$$
F_{n}(\omega)=\int_{0}^{2 \pi} F_{n}(\omega, \theta) \cos n \theta \mathrm{d} \theta
$$

A general Kolmogorov spectrum is defined by the following system of equations:

$$
\begin{aligned}
& P+\omega Q=\int_{0}^{\omega}\left(\omega-\omega_{1}\right) F_{0}\left(\omega_{1}\right) \mathrm{d} \omega_{1} \\
& M=\frac{1}{g} \int_{0}^{\omega} \omega_{1}^{2} F_{1}\left(\omega_{1}\right) \mathrm{d} \omega_{1} \\
& F_{n}(\omega)=0 \quad \text { if } n \geq 2
\end{aligned}
$$

Now $\varepsilon_{\omega}(\theta)=\omega N_{\omega}(\theta)$. One can present $N$ in a form of the Fourier series

$$
N(\omega, \theta)=\frac{1}{2 \pi} \sum N_{n}(\omega) \cos n \theta
$$

and turn (C.5)-(C.7) into an infinite system of nonlinear integral equations imposed on $N_{n}(\omega)$.

\section{References}

[1] M.L. Banner, Equilibrium spectra of wind waves, J. Phys. Oceanogr. 20 (1990) 966-984.

[2] V.E. Zakharov, G. Falkovich, V. Lvov, Kolmogorov Spectra of Turbulence, vol. I, Springer, Berlin, 1992. 
[3] A.N. Pushkarev, V.E. Zakharov, Turbulence of capillary waves, Phys. Rev. Lett. 76 (1996) 3320-3323.

[4] A.N. Pushkarev, V.E. Zakharov, Turbulence of capillary waves-theory and numerical simulation, Physica D 135 (2000) 98.

[5] V.E. Zakharov, N.N. Filonenko, Weak turbulence of capillary waves, J. Appl. Mech. Tech. Phys. 4 (1967) 506-515.

[6] V.E. Zakharov, N.N. Filonenko, The energy spectrum for stochastic oscillation of a fluid surface, Dokl. Acad. Nauk SSSR 170 (1966) 1292-1295.

[7] K. Hasselmann, On the nonlinear energy transfer in a gravity-wave spectrum. Part I. General theory, J. Fluid Mech. 12 (1962) $481-500$.

[8] K. Hasselmann, On the nonlinear energy transfer in a gravity wave spectrum. Part 2. Conservative theorems, wave-particle analogy, irreversability, J. Fluid Mech. 15 (1963) 273-281.

[9] M.A. Donelan, J. Hamilton, W.H. Hui, Directional spectra of wind-generated waves, Phil. Trans. R. Soc. London A 315 (1985) $509-562$.

[10] A. Newell, V.E. Zakharov, Rough sea phoam, Phys .Rev. Lett. 69 (1992) 1149-1151.

[11] Y. Toba, Local balance in the air-sea boundary processes on the spectrum of wind waves, J. Oceanogr. Soc. Jpn. 29 (1973) $209-220$.

[12] V.E. Zakharov, M.M. Zaslavsky, The kinetic equation and Kolmogorov spectra in the weak-turbulence theory of wind waves, Izv. Atm. Ocean. Phys. 18 (1982) 747-753.

[13] S.A. Kitaigorodskii, On the theory of the equilibrium range in the spectrum of wind-generated gravity waves, J. Phys. Oceanogr. 13 (1983) 816-827.

[14] V.E. Zakharov, A.N. Pushkarev, Diffusion model of interacting gravity waves on the surface of deep liquid, Nonlin. Process. Geophys. 6 (1999) $1-10$.

[15] A.N. Pushkarev, V.E. Zakharov, in: Proceedings of the Sixth International Workshop on Wave Hindcasting and Forecasting, Preprints, Monterey, CA, 2000.

[16] S. Hasselmann, K. Hasselmann, T.P. Barnett, Computation and parametrization of the nonlinear energy transfer in gravity wave spectrum, J. Phys. Oceanogr. 15 (1985) 1378-1391.

[17] J.C. Dungey, W.H. Hui, Nonlinear energy transfer in a narrow gravity-wave spectrum, Proc. R. Soc. A 368 (1985) $239-265$.

[18] A. Masuda, Nonlinear energy transfer between wind waves, J. Phys. Oceanogr. 10 (1981) 2082-2093.

[19] A. Masuda, in: O.M. Phillips, K. Hasselmann (Eds.), Waves Dynamics and Radio Probing of the Ocean Surface, Plenum Press, New York, 1986.

[20] I.V. Lavrenov, Mathematical Modeling of Wind Waves at Non-uniform Ocean, Gidrometeoizdat, St. Petersburg, 1998.

[21] V.G. Polnikov, Numerical modeling of the constant flux spectra for surface gravity waves in the case of angular anysotropy, Wave Motion 1008 (2001) 1-12.

[22] D.J. Webb, Nonlinear transfer between weak waves, Deep-Sea Res. 25 (1978) 279-298.

[23] D. Resio, W. Perrie, A numerical study of nonlinear fluxes due to wave-wave interaction. Part 1. Methodology and basic resuts, J. Fluid Mech. 223 (1991) 603-629.

[24] P.A. Hwang, et al., Airborne measurements of the wavenumber spectra of ocean surface waves. Part I. Spectral slope and dimensionless spectral coefficient, J. Phys. Oceanogr. 30 (2000) 2753-2767.

[25] I.R. Young, L.A. Verhagen, M.L. Banner, A note on the bi-modal directional spreading of fetch-limited wind waves, J. Geophys. Res. 100 (1995) 773-778.

[26] J.Z. Forristall, Measurements of a saturated range in ocean wave spectra, J. Geophys. Res. 86 (1981) 8075-8084.

[27] K.K. Kahma, A study of the growth of the wave spectrum with fetch, J. Phys. Oceanogr. 11 (1981) 1503-1515.

[28] S. Kawai, K. Okuda, Y. Toba, Field data support of three-second power law and $g u_{\star} \sigma^{-4}$ spectral form for growing wind waves, J. Oceanogr. Soc. Jpn. 33 (1977) 137-150.

[29] H. Mitsuyasu, et al., Observation of the power spectrum of ocean waves using clover-leaf buoy, J. Phys. Oceanogr. 10 (1980) $286-296$.

[30] O.M. Phillips, Spectral and statistical properties of the equilibrium range in wind-generated gravity waves, J. Fluid Mech. 156 (1985) $505-531$.

[31] J.A. Battjes, T.J. Zitman, L.H. Holthuijsen, A re-analysis of the spectra observed in JONSWAP, J. Phys. Oceanogr. 17 (1987) $1288-1295$.

[32] W. Perrie, V. Zakharov, The equilibrium range cascades of wind-generated waves, Eur. J. Mech. B 18 (1999) 365-371.

[33] V.E. Zakharov, Statistical theory of gravity and capillary waves on the surface of a finite-depth fluid, Eur. J. Mech. B 18 (1999) $327-344$.

[34] V.E. Zakharov, Inverse and direct cascade in a wind-driven surface wave turbulence and wave-breaking, in: Proceedings of the IUTAM Symposium, Sydney, Australia, Springer, 1992.

[35] G.J. Komen, S. Hasselmann, K. Hasselmann, On the existence of a fully developed wind-sea spectrum, J. Phys. Oceanogr. 14 (1984) $1271-1285$.

[36] M.L. Banner, I.R. Young, Modelling spectral dissipation in the evolution of wind-waves. Part 1. Assessment of existing model performance, J. Phys. Oceanogr. 24 (1994) 1550-1571.

[37] O.M. Phillips, The equilibrium range in the spectrum of wind-generated waves, J. Fluid Mech. 4 (1958) $426-434$.

[38] W.J. Pierson, L.A. Moskowitz, A proposed spectral form for fully developed wind seas based on similarity theory of S.A. Kitaigorodskii, J. Geophys. Res. 69 (1964) 5181-5190.

[39] K. Hasselmann, et al., Measurements of wind-wave growth and swell decay during JONSWAP, Dtsch. Hydrogr. Z. 12 (Suppl. A8) (1973) 95.

[40] D. Resio, J.H. Pihl, B.A. Tracy, C.L. Vincent, Nonlinear energy fluxes and the finite depth equilibrium range in wave spectra, J. Geophys. Res. 106 (2001) 6985-7000.

[41] I.R. Young, Wind Generated Ocean Waves, Elsevier, Amsterdam, 1999. 